\title{
Divisibility properties of sporadic Apéry-like numbers
}

\author{
Amita Malik ${ }^{1}$ and Armin Straub $1,2^{*}$
}

\author{
*Correspondence: \\ straub@southalabama.edu \\ 1 Department of Mathematics, \\ University of Illinois at \\ Urbana-Champaign, 1409 W Green \\ St, Urbana, IL 61801, USA \\ 2 Department of Mathematics and \\ Statistics, University of South \\ Alabama, 411 University Blvd N \\ Mobile, AL 36688, USA
}

\begin{abstract}
In 1982, Gessel showed that the Apéry numbers associated to the irrationality of $\zeta$ (3) satisfy Lucas congruences. Our main result is to prove corresponding congruences for all known sporadic Apéry-like sequences. In several cases, we are able to employ approaches due to Mclntosh, Samol-van Straten and Rowland-Yassawi to establish these congruences. However, for the sequences labeled $s_{18}$ and $(\eta)$ we require a finer analysis.

As an application, we investigate modulo which numbers these sequences are periodic. In particular, we show that the Almkvist-Zudilin numbers are periodic modulo 8, a special property which they share with the Apéry numbers. We also investigate primes which do not divide any term of a given Apéry-like sequence.
\end{abstract}

Keywords: Apéry-like numbers, Lucas congruences, $p$-adic properties

\section{Introduction}

In his surprising proof $[5,24]$ of the irrationality of $\zeta(3), R$. Apéry introduced the sequence

$$
A(n)=\sum_{k=0}^{n}\left(\begin{array}{l}
n \\
k
\end{array}\right)^{2}\left(\begin{array}{c}
n+k \\
k
\end{array}\right)^{2},
$$

which has since been referred to as the Apéry sequence. It was shown by I. Gessel ([16], Theorem 1) that, for any prime $p$, these numbers satisfy the Lucas congruences

$$
A(n) \equiv A\left(n_{0}\right) A\left(n_{1}\right) \cdots A\left(n_{r}\right) \quad(\bmod p),
$$

where $n=n_{0}+n_{1} p+\cdots+n_{r} p^{r}$ is the expansion of $n$ in base $p$. Initial work of F. Beukers [8] and D. Zagier [29], which was extended by G. Almkvist, W. Zudilin [4] and S. Cooper [12], has complemented the Apéry numbers with a, conjecturally finite, set of sequences, known as Apéry-like, which share (or are believed to share) many of the remarkable properties of the Apéry numbers, such as connections to modular forms [2, 7, 27] or supercongruences $[6,10,13,21-23]$. After briefly reviewing Apéry-like sequences in Section 2, we prove in Sections 3 and 4 our main result that all of these sequences also satisfy the Lucas congruences (1.2). For all but two of the sequences, we establish these congruences in Section 3 by extending a general approach provided by R. McIntosh [19]. The main difficulty, however, lies in establishing these congruences for the sequence $(\eta)$. For this sequence, and to a lesser extent for the sequence $s_{18}$, we require a much finer analysis, which is given separately in Section 4.

(c) 2016 Malik and Straub. Open Access This article is distributed under the terms of the Creative Commons Attribution 4.0 International License (http://creativecommons.org/licenses/by/4.0/), which permits unrestricted use, distribution, and reproduction in any medium, provided you give appropriate credit to the original author(s) and the source, provide a link to the Creative Commons license, and indicate if changes were made. 
In the approaches of Gessel and McIntosh, binomial sums, like (1.1), are used to derive Lucas congruences. Other known approaches to proving Lucas congruences for a sequence $C(n)$ are based on expressing $C(n)$ as the constant terms of powers of a Laurent polynomial or as the diagonal coefficients of a multivariate algebraic function. However, neither of these approaches is known to apply, for instance, to the sequence $(\eta)$. In the first approach, one seeks a Laurent polynomial $\Lambda(x)=\Lambda\left(x_{1}, \ldots, x_{d}\right)$ such that $C(n)$ is the constant term of $\Lambda(\boldsymbol{x})$. In that case, we write $C(n)=$ ct $\Lambda(\boldsymbol{x})^{n}$ for brevity. If the Newton polyhedron of $\Lambda(\boldsymbol{x})$ has the origin as its only interior integral point, the results of K. Samol and D. van Straten [26] (see also [20]) apply to show that $C(n)$ satisfies the Dwork congruences

$$
C\left(p^{r} m+n\right) C(\lfloor n / p\rfloor) \equiv C\left(p^{r-1} m+\lfloor n / p\rfloor\right) C(n) \quad\left(\bmod p^{r}\right)
$$

for all primes $p$ and all integers $m, n \geq 0, r \geq 1$. The case $r=1$ of these congruences is equivalent to the Lucas congruences (1.2) for the sequence $C(n)$. For instance, in the case of the Apéry numbers (1.1), we have ([28], Remark 1.4)

$$
A(n)=\operatorname{ct}\left[\frac{(x+y)(z+1)(x+y+z)(y+z+1)}{x y z}\right]^{n},
$$

from which one may conclude that the Apéry numbers satisfy the congruences (1.3), generalizing (1.2). Similarly, for the sequence $(\eta)$, one may derive from the binomial sum (4.2), using G. Egorychev's method of coefficients [15], that its $n$th term is given by ct $\Lambda(x, y, z)^{n}$, where

$$
\Lambda(x, y, z)=\left(1-\frac{1}{x y(1+z)^{5}}\right) \frac{(1+x)(1+y)(1+z)^{4}}{z^{3}} .
$$

However, $\Lambda(x, y, z)$ is not a Laurent polynomial, and it is unclear if and how one could express the sequence $(\eta)$ as constant terms of powers of an appropriate Laurent polynomial. As a second general approach, E. Rowland and R. Yassawi [25] show that Lucas congruences hold for a certain class of sequences that can be represented as the diagonal Taylor coefficients of $1 / Q(\boldsymbol{x})^{1 / s}$, where $s \geq 1$ is an integer and $Q(\boldsymbol{x}) \in \mathbb{Z}[\boldsymbol{x}]$ is a multivariate polynomial. Again, while such representations are known for some Apérylike sequences, see, for instance, [28], no suitable representations are available for the sequences $(\eta)$ or $s_{18}$.

It was conjectured by S. Chowla, J. Cowles and M. Cowles [11] and subsequently proven by I. Gessel [16] that

$$
A(n) \equiv\left\{\begin{array}{l}
1, \text { if } n \text { is even, } \\
5, \text { if } n \text { is odd, }
\end{array}(\bmod 8) .\right.
$$

The congruences (1.4) show that the Apéry numbers are periodic modulo 8, and it was recently demonstrated by E. Rowland and R. Yassawi [25] that they are not eventually periodic modulo 16, thus answering a question of Gessel. The Apéry numbers are also periodic modulo 3 (see (5.1)) and their values modulo 9 are characterized by an extension of the Lucas congruences [16]; see also the recent generalizations [17] of C. Krattenthaler and T. Müller, who characterize generalized Apéry numbers modulo 9. As an application of the Lucas congruences established in Sections 3 and 4, we address in Section 5 the natural question to which extent results like (1.4) are true for Apéry-like numbers in general. In particular, we show in Theorem 5.3 that the Almkvist-Zudilin numbers are periodic modulo 8 as well. 
The primes 2, 3, 7, 13, 23, 29, 43, 47, .. do not divide any Apéry number $A(n)$, and E. Rowland and R. Yassawi [25] pose the question whether there are infinitely many such primes. While this question remains open, we offer numerical and heuristic evidence that a positive proportion of the primes, namely, about $e^{-1 / 2} \approx 60.65 \%$, do not divide any Apéry number. In Section 6, we investigate the analogous question for other Apéry-like numbers, and prove that Cooper's sporadic sequences [12] behave markedly differently. Indeed, for any given prime $p$, a fixed proportion of the last of the first $p$ terms of these sequences is divisible by $p$. In the case of sums of powers of binomial coefficients, such a result has been proven by N. Calkin [9].

\section{Review of Apéry-like numbers}

Along with the Apéry numbers $A(n)$, defined in (1.1), R. Apéry also introduced the sequence

$$
B(n)=\sum_{k=0}^{n}\left(\begin{array}{l}
n \\
k
\end{array}\right)^{2}\left(\begin{array}{c}
n+k \\
k
\end{array}\right),
$$

which allowed him to (re)prove the irrationality of $\zeta(2)$. This sequence is the solution of the three-term recursion

$$
(n+1)^{2} u_{n+1}=\left(a n^{2}+a n+b\right) u_{n}-c n^{2} u_{n-1},
$$

with the choice of parameters $(a, b, c)=(11,3,-1)$ and initial conditions $u_{-1}=0, u_{0}=$ 1 . Because we divide by $(n+1)^{2}$ at each step, it is not to be expected that the recursion (2.1) should have an integer solution. Inspired by F. Beukers [8], D. Zagier [29] conducted a systematic search for other choices of the parameters $(a, b, c)$ for which the solution to (2.1), with initial conditions $u_{-1}=0, u_{0}=1$, is integral. After normalizing, and apart from degenerate cases, he discovered four hypergeometric, four Legendrian as well as six sporadic solutions. It is still open whether further solutions exist or even that there should be only finitely many solutions. The six sporadic solutions are reproduced in Table 1 . Note that each binomial sum included in this table certifies that the corresponding sequence indeed consists of integers.

Similarly, the Apéry numbers $A(n)$, defined in (1.1), are the solution of the three-term recurrence

$$
(n+1)^{3} u_{n+1}=(2 n+1)\left(a n^{2}+a n+b\right) u_{n}-n\left(c n^{2}+d\right) u_{n-1}
$$

Table 1 The six sporadic solutions of (2.1) and their labels in [29] and [3]

\begin{tabular}{llll}
\hline$(a, b, c)$ & {$[29]$} & {$[3]$} & $A(n)$ \\
\hline$(7,2,-8)$ & $\mathbf{A}$ & $(\mathrm{a})$ & $\sum_{k}\left(\begin{array}{l}n \\
k\end{array}\right)^{3}$ \\
$(11,3,-1)$ & $\mathbf{D}$ & $(\mathrm{b})$ & $\sum_{k}\left(\begin{array}{l}n \\
k\end{array}\right)^{2}\left(\begin{array}{c}n+k \\
n\end{array}\right)$ \\
$(10,3,9)$ & $\mathbf{C}$ & $(\mathrm{c})$ & $\sum_{k}\left(\begin{array}{l}n \\
k\end{array}\right)^{2}\left(\begin{array}{c}2 k \\
k\end{array}\right)$ \\
$(12,4,32)$ & $\mathbf{E}$ & $(\mathrm{d})$ & $\sum_{k}\left(\begin{array}{l}n \\
k\end{array}\right)\left(\begin{array}{c}2 k \\
k\end{array}\right)\left(\begin{array}{c}2(n-k) \\
n-k\end{array}\right)$ \\
$(9,3,27)$ & $\mathbf{B}$ & $(\mathrm{f})$ & $\sum_{k}(-1)^{k} 3^{n-3 k}\left(\begin{array}{l}n \\
3 k\end{array}\right) \frac{(3 k) !}{k ! 3}$ \\
$(17,6,72)$ & $\mathbf{F}$ & $(\mathrm{g})$ & $\sum_{k, l}(-1)^{k} 8^{n-k}\left(\begin{array}{l}n \\
k\end{array}\right)\left(\begin{array}{l}k \\
l\end{array}\right)$ \\
\hline
\end{tabular}


with the choice of parameters $(a, b, c, d)=(17,5,1,0)$ and initial conditions $u_{-1}=0$, $u_{0}=1$. Systematic computer searches for further integer solutions have been performed by G. Almkvist and W. Zudilin [4] in the case $d=0$ and, more recently, by S. Cooper [12], who introduced the additional parameter $d$. As in the case of (2.1), apart from degenerate cases, only finitely many sequences have been discovered. In the case $d=0$, there are again six sporadic sequences, which are recorded in Table 2. Moreover, by general principles (see [12] Eq. (17)), each of the sequences in Table 1 times $\left(\begin{array}{c}2 n \\ n\end{array}\right)$ is an integer solution of (2.2) with $d \neq 0$. Apart from such expected solutions, Cooper also found three additional sporadic solutions, including

$$
s_{18}(n)=\sum_{k=0}^{\lfloor n / 3\rfloor}(-1)^{k}\left(\begin{array}{l}
n \\
k
\end{array}\right)\left(\begin{array}{c}
2 k \\
k
\end{array}\right)\left(\begin{array}{c}
2(n-k) \\
n-k
\end{array}\right)\left[\left(\begin{array}{c}
2 n-3 k-1 \\
n
\end{array}\right)+\left(\begin{array}{c}
2 n-3 k \\
n
\end{array}\right)\right],
$$

for $n \geq 1$, with $s_{18}(0)=1$, as well as $s_{7}$ and $s_{10}$, which are included in Table 2. Remarkably, these sequences are again connected to modular forms [12] (the subscript refers to the level) and satisfy supercongruences, which are proved in [23]. Indeed, it was the corresponding modular forms and Ramanujan-type series for $1 / \pi$ that led Cooper to study these sequences, and the binomial expressions for $s_{7}$ and $s_{18}$ were found subsequently by Zudilin (sequence $s_{10}$ was well-known before).

\section{Lucas congruences}

It is a well-known and beautiful classical result of Lucas [18] that the binomial coefficients satisfy the congruences

$$
\left(\begin{array}{l}
n \\
k
\end{array}\right) \equiv\left(\begin{array}{l}
n_{0} \\
k_{0}
\end{array}\right)\left(\begin{array}{l}
n_{1} \\
k_{1}
\end{array}\right) \cdots\left(\begin{array}{l}
n_{r} \\
k_{r}
\end{array}\right) \quad(\bmod p),
$$

where $p$ is a prime and $n_{i}$, respectively $k_{i}$, are the $p$-adic digits of $n$ and $k$. That is, $n=$ $n_{0}+n_{1} p+\cdots+n_{r} p^{r}$ and $k=k_{0}+k_{1} p+\cdots+k_{r} p^{r}$ are the expansions of $n$ and $k$ in base $p$. Correspondingly, a sequence $a(n)$ is said to satisfy Lucas congruences, if the congruences

$$
a(n) \equiv a\left(n_{0}\right) a\left(n_{1}\right) \cdots a\left(n_{r}\right) \quad(\bmod p)
$$

\begin{tabular}{|c|c|c|c|}
\hline$(a, b, c, d)$ & [3] & [12] & $A(n)$ \\
\hline$(7,3,81,0)$ & $(\delta)$ & & $\sum_{k}(-1)^{k} 3^{n-3 k}\left(\begin{array}{c}n \\
3 k\end{array}\right)\left(\begin{array}{c}n+k \\
n\end{array}\right) \frac{(3 k) !}{k !^{3}}$ \\
\hline$(11,5,125,0)$ & $(\eta)$ & & $\sum_{k=0}(-1)^{k}\left(\begin{array}{l}n \\
k\end{array}\right)^{3}\left(\left(\begin{array}{c}4 n-5 k-1 \\
3 n\end{array}\right)+\left(\begin{array}{c}4 n-5 k \\
3 n\end{array}\right)\right)$ \\
\hline$(10,4,64,0)$ & $(\alpha)$ & & $\sum_{k}\left(\begin{array}{l}n \\
k\end{array}\right)^{2}\left(\begin{array}{c}2 k \\
k\end{array}\right)\left(\begin{array}{c}2(n-k) \\
n-k\end{array}\right)$ \\
\hline$(12,4,16,0)$ & $(\epsilon)$ & & $\sum_{k}\left(\begin{array}{l}n \\
k\end{array}\right)^{2}\left(\begin{array}{c}2 k \\
n\end{array}\right)^{2}$ \\
\hline$(9,3,-27,0)$ & $(\zeta)$ & & $\sum_{k, l}\left(\begin{array}{l}n \\
k\end{array}\right)^{2}\left(\begin{array}{l}n \\
l\end{array}\right)\left(\begin{array}{l}k \\
l\end{array}\right)\left(\begin{array}{c}k+l \\
n\end{array}\right)$ \\
\hline$(17,5,1,0)$ & $(\gamma)$ & & $\sum_{k}\left(\begin{array}{l}n \\
k\end{array}\right)^{2}\left(\begin{array}{c}n+k \\
n\end{array}\right)^{2}$ \\
\hline$(13,4,-27,3)$ & & $s_{7}$ & $\sum_{k}^{n}\left(\begin{array}{l}n \\
k\end{array}\right)^{2}\left(\begin{array}{c}n+k \\
k\end{array}\right)\left(\begin{array}{c}2 k \\
n\end{array}\right)$ \\
\hline$(6,2,-64,4)$ & & $s_{10}$ & $\sum_{k}\left(\begin{array}{l}n \\
k\end{array}\right)^{4}$ \\
\hline$(14,6,192,-12)$ & & $s_{18}$ & defined in (2.3) \\
\hline
\end{tabular}

Table 2 The sporadic solutions of (2.2) 
hold for all primes $p$. It was shown by I. Gessel ([16], Theorem 1) that the Apéry numbers $A(n)$, defined in (1.1), satisfy Lucas congruences. E. Deutsch and B. Sagan ([14], Theorem 5.9) show that the Lucas congruences (3.2) in fact hold for the family of generalized Apéry sequences

$$
A_{r, s}(n)=\sum_{k=0}^{n}\left(\begin{array}{l}
n \\
k
\end{array}\right)^{r}\left(\begin{array}{c}
n+k \\
k
\end{array}\right)^{s},
$$

with $r$ and $s$ positive integers. This family includes the sequences (a), (b) from Table 1, and the sequences $(\gamma), s_{10}$ from Table 2 . The purpose of this section and Section 4 is to show that, in fact, all the Apéry-like sequences in Tables 1 and 2 satisfy the Lucas congruences (3.2). Using and extending the general framework provided by R. McIntosh ([19], Theorem 6), which we review below, we are able to prove this claim for all of the sequences in the two tables, with the exception of the two sequences $(\eta)$ and $s_{18}$, for which we require a much finer analysis, which is given in Section 4.

Theorem 3.1. Each of the sequences from Tables 1 and 2 satisfies the Lucas congruences (3.2).

Remark 3.2. The Lucas congruences (3.2), in general, do not extend to prime powers. However, it is shown in [16], and generalized in [17], that the Lucas congruences modulo 3 for the Apéry numbers extend to hold modulo 9.

On the other hand, numerical evidence suggests that all the Apéry-like sequences from Tables 1 and 2 in fact satisfy the Dwork congruences (1.3). While Theorem 3.1 proves the case $r=1$ of these congruences, it would be desirable to establish the corresponding congruences modulo higher powers of primes.

Following [19], we say that a function $L: \mathbb{Z}_{\geq 0}^{2} \rightarrow \mathbb{Z}$ has the double Lucas property (DLP) if $L(n, k)=0$, for $k>n$, and if

$$
L(n, k) \equiv L\left(n_{0}, k_{0}\right) L\left(n_{1}, k_{1}\right) \cdots L\left(n_{r}, k_{r}\right) \quad(\bmod p),
$$

for every prime $p$. Here, as in (3.1), $n_{i}$ and $k_{i}$ are the $p$-adic digits of $n$ and $k$, respectively. Eq. (3.1) shows that the binomial coefficients $\left(\begin{array}{l}n \\ k\end{array}\right)$ are a DLP function. More generally, it is shown in ([19], Theorem 6) that, for positive integers $r_{0}, r_{1}, \ldots, r_{m}$,

$$
L(n, k)=\left(\begin{array}{l}
n \\
k
\end{array}\right)^{r_{0}}\left(\begin{array}{c}
n+k \\
k
\end{array}\right)^{r_{1}}\left(\begin{array}{c}
n+2 k \\
k
\end{array}\right)^{r_{2}} \cdots\left(\begin{array}{c}
n+m k \\
k
\end{array}\right)^{r_{m}}
$$

is a DLP function. For instance, choosing the exponents as $r_{i}=1$, we find that the multinomial coefficient

$$
\left(\begin{array}{c}
n+m k \\
k, k, \ldots, k, n-k
\end{array}\right)=\frac{(n+m k) !}{k !^{m+1}(n-k) !}
$$

is a DLP function for any integer $m \geq 0$.

Suppose that $L(n, k)$ is a DLP function and that $G(n)$ and $H(n)$ are $\mathbf{L P}$ functions, that is, the sequences $G(n)$ and $H(n)$ satisfy the Lucas congruences (3.2). Then, as shown in ([19], Theorem 5),

$$
F(n)=\sum_{k=0}^{n} L(n, k) G(k) H(n-k)
$$


is an LP function. Note that (3.5) and (3.6) combined are already sufficient to prove that the generalized Apéry sequences, defined in (3.3), satisfy Lucas congruences. In order to apply this machinery more generally, and prove Theorem 3.1, our next results extend the repertoire of DLP functions. In fact, it turns out that we need a natural extension of the Lucas property to the case of three variables. We say that a function $M: \mathbb{Z}_{\geq 0}^{3} \rightarrow \mathbb{Z}$ has the triple Lucas property (TLP) if $M(n, k, j)=0$, for $j>n$, and if

$$
M(n, k, j) \equiv M\left(n_{0}, k_{0}, j_{0}\right) \cdots M\left(n_{r}, k_{r}, j_{r}\right) \quad(\bmod p),
$$

for every prime $p$, where $n_{i}, k_{i}$ and $j_{i}$ are the $p$-adic digits of $n, k$ and $j$, respectively. It is straightforward to prove the following analog of (3.6) for TLP functions.

Lemma 3.3. If $M(n, k, j)$ is a TLP function, then

$$
L(n, k)=\sum_{j=0}^{n} M(n, k, j)
$$

satisfies the double Lucas congruences (3.4). In particular, if $L(n, k)=0$, for $k>n$, then $L(n, k)$ is a DLP function.

Proof. Let $p$ be a prime. It is enough to show that, for any nonnegative integers $n_{0}, n^{\prime}, k_{0}, k^{\prime}$ such that $n_{0}<p$ and $k_{0}<p$,

$$
L\left(n_{0}+n^{\prime} p, k_{0}+k^{\prime} p\right) \equiv L\left(n_{0}, k_{0}\right) L\left(n^{\prime}, k^{\prime}\right) \quad(\bmod p) .
$$

Since the sum defining $L(n, k)$ is naturally supported on $j \in\{0,1, \ldots, n\}$, we may extend it over all $j \in \mathbb{Z}$. Modulo $p$, we have

$$
\begin{aligned}
L(n, k) & =\sum_{j \in \mathbb{Z}} M(n, k, j) \\
& =\sum_{j_{0}=0}^{p-1} \sum_{j^{\prime} \in \mathbb{Z}} M\left(n, k, j_{0}+j^{\prime} p\right) \\
& \equiv \sum_{j_{0} \in \mathbb{Z} j^{\prime} \in \mathbb{Z}} M\left(n_{0}, k_{0}, j_{0}\right) M\left(n^{\prime}, k^{\prime}, j^{\prime}\right) \\
& =L\left(n_{0}, k_{0}\right) L\left(n^{\prime}, k^{\prime}\right),
\end{aligned}
$$

which is what we had to prove.

Lemma 3.4. The function

$$
M(n, k, j)=\left(\begin{array}{c}
n \\
j
\end{array}\right)\left(\begin{array}{c}
k+j \\
n
\end{array}\right)
$$

is a TLP function.

Proof. Clearly, $M(n, k, j)=0$, for $j>n$. In order to show that $M(n, k, j)$ is a TLP function, we therefore need to show that, for any prime $p$,

$$
M\left(n_{0}+n^{\prime} p, k_{0}+k^{\prime} p, j_{0}+j^{\prime} p\right) \equiv M\left(n_{0}, k_{0}, j_{0}\right) M\left(n^{\prime}, k^{\prime}, j^{\prime}\right) \quad(\bmod p),
$$

provided that $0 \leq n_{0}, k_{0}, j_{0}<p$ and $n^{\prime}, k^{\prime}, j^{\prime} \geq 0$. Observe that in the case $j_{0}>n_{0}$ both sides of the congruence (3.9) vanish because of the Lucas congruences (3.1) for the binomial coefficients. We may therefore proceed under the assumption that $j_{0} \leq n_{0}$. 
Writing $\left[x^{n}\right] f(x)$ for the coefficient of $x^{n}$ in the polynomial $f(x)$, we begin with the simple observation that

$$
\left(\begin{array}{c}
k+j \\
n
\end{array}\right)=\left[x^{n}\right](1+x)^{k+j} .
$$

Modulo $p$, we have

$$
(1+x)^{k+j}=(1+x)^{k_{0}+j_{0}}(1+x)^{\left(k^{\prime}+j^{\prime}\right) p} \equiv(1+x)^{k_{0}+j_{0}}\left(1+x^{p}\right)^{k^{\prime}+j^{\prime}} \quad(\bmod p) .
$$

Since $0 \leq k_{0}+j_{0}<2 p$, extracting the coefficient of $x^{n}=x^{n_{0}}\left(x^{p}\right)^{n^{\prime}}$ from this product results in the congruence

$$
\left(\begin{array}{c}
k+j \\
n
\end{array}\right) \equiv\left(\begin{array}{c}
k_{0}+j_{0} \\
n_{0}
\end{array}\right)\left(\begin{array}{c}
k^{\prime}+j^{\prime} \\
n^{\prime}
\end{array}\right)+\left(\begin{array}{c}
k_{0}+j_{0} \\
n_{0}+p
\end{array}\right)\left(\begin{array}{c}
k^{\prime}+j^{\prime} \\
n^{\prime}-1
\end{array}\right) \quad(\bmod p) .
$$

Note that, under our assumption that $j_{0} \leq n_{0}$, the second term on the right-hand side of this congruence vanishes (since $n_{0}+p \geq j_{0}+p>j_{0}+k_{0}$ ). This, along with (3.1), proves (3.9).

Corollary 3.5. The function

$$
L(n, k)=\left(\begin{array}{l}
n \\
k
\end{array}\right)\left(\begin{array}{c}
2 k \\
n
\end{array}\right)
$$

is a DLP function.

Proof. Set $j=k$ in Lemma 3.4.

Lemma 3.6. The function

$$
L(n, k)=3^{n-3 k}\left(\begin{array}{c}
n \\
3 k
\end{array}\right) \frac{(3 k) !}{k !^{3}}
$$

is a DLP function.

Proof. Let $p$ be a prime. As usual, we write $n=n_{0}+n^{\prime} p$ and $k=k_{0}+k^{\prime} p$ where $0 \leq n_{0}<p$ and $0 \leq k_{0}<p$. In light of (3.1) and (3.6), the simple observation

$$
\left(\begin{array}{c}
2 n \\
n
\end{array}\right)=\sum_{k=0}^{n}\left(\begin{array}{l}
n \\
k
\end{array}\right)^{2},
$$

demonstrates that the sequence of central binomial coefficients is an LP function. We claim that

$$
\frac{(3 k) !}{k !^{3}}=\left(\begin{array}{c}
3 k \\
k
\end{array}\right)\left(\begin{array}{c}
2 k \\
k
\end{array}\right)
$$

is an $\mathbf{L P}$ function as well. From the Lucas congruences for the central binomials, that is

$$
\left(\begin{array}{c}
2 k \\
k
\end{array}\right) \equiv\left(\begin{array}{c}
2 k_{0} \\
k_{0}
\end{array}\right)\left(\begin{array}{c}
2 k^{\prime} \\
k^{\prime}
\end{array}\right) \quad(\bmod p)
$$

we observe that $\left(\begin{array}{c}2 k \\ k\end{array}\right)$ is divisible by $p$ if $2 k_{0} \geq p$. Hence, we only need to show the congruences

$$
\frac{(3 k) !}{k !^{3}} \equiv \frac{\left(3 k_{0}\right) !}{k_{0} !^{3}} \frac{\left(3 k^{\prime}\right) !}{k^{\prime} !^{3}}(\bmod p)
$$


under the assumption that $k_{0}<p / 2$. Note that

$$
\begin{aligned}
\left(\begin{array}{c}
3 k \\
k
\end{array}\right) & =\left[x^{k}\right](1+x)^{3 k} \\
& \equiv\left[x^{k_{0}}\left(x^{p}\right)^{k^{\prime}}\right](1+x)^{3 k_{0}}\left(1+x^{p}\right)^{3 k^{\prime}} \quad(\bmod p) \\
& =\left(\begin{array}{c}
3 k_{0} \\
k_{0}
\end{array}\right)\left(\begin{array}{c}
3 k^{\prime} \\
k^{\prime}
\end{array}\right)+\left(\begin{array}{c}
3 k_{0} \\
k_{0}+p
\end{array}\right)\left(\begin{array}{c}
3 k^{\prime} \\
k^{\prime}-1
\end{array}\right)+\left(\begin{array}{c}
3 k_{0} \\
k_{0}+2 p
\end{array}\right)\left(\begin{array}{c}
3 k^{\prime} \\
k^{\prime}-2
\end{array}\right) .
\end{aligned}
$$

In the case $k_{0}<p / 2$, we have $k_{0}+p>3 k_{0}$, so that the last two terms on the right-hand side vanish. This proves (3.11).

Next, we claim that

$$
\left(\begin{array}{c}
n \\
3 k
\end{array}\right) \frac{(3 k) !}{k !^{3}} \equiv\left(\begin{array}{c}
n_{0} \\
3 k_{0}
\end{array}\right) \frac{\left(3 k_{0}\right) !}{k_{0} !^{3}}\left(\begin{array}{c}
n^{\prime} \\
3 k^{\prime}
\end{array}\right) \frac{\left(3 k^{\prime}\right) !}{k^{\prime} !^{3}} \quad(\bmod p) .
$$

By congruence (3.11), both sides vanish modulo $p$ if $3 k_{0} \geq p$. On the other hand, if $3 k_{0}<p$, then the usual argument shows that

$$
\left(\begin{array}{c}
n \\
3 k
\end{array}\right) \equiv\left[x^{3 k_{0}}\left(x^{p}\right)^{3 k^{\prime}}\right](1+x)^{n_{0}}\left(1+x^{p}\right)^{n^{\prime}}=\left(\begin{array}{c}
n_{0} \\
3 k_{0}
\end{array}\right)\left(\begin{array}{c}
n^{\prime} \\
3 k^{\prime}
\end{array}\right) \quad(\bmod p) .
$$

In combination with (3.11), this proves (3.12).

Finally, the congruences $L(n, k) \equiv L\left(n_{0}, k_{0}\right) L\left(n^{\prime}, k^{\prime}\right)$, that is

$$
3^{n-3 k}\left(\begin{array}{c}
n \\
3 k
\end{array}\right) \frac{(3 k) !}{k !^{3}} \equiv 3^{n_{0}-3 k_{0}}\left(\begin{array}{c}
n_{0} \\
3 k_{0}
\end{array}\right) \frac{\left(3 k_{0}\right) !}{k_{0} !^{3}} 3^{n^{\prime}-3 k^{\prime}}\left(\begin{array}{c}
n^{\prime} \\
3 k^{\prime}
\end{array}\right) \frac{\left(3 k^{\prime}\right) !}{k^{\prime} !^{3}} \quad(\bmod p),
$$

follow from Fermat's little theorem and the fact that both sides vanish if $3 k_{0}>n_{0}$ or $3 k^{\prime}>n^{\prime}$.

We are now in a comfortable position to prove Theorem 3.1 for all but two of the sporadic Apéry-like sequences. To show that sequences $(\eta)$ and $s_{18}$ satisfy Lucas congruences as well requires considerable additional effort, and the corresponding proofs are given in Section 4.

Proof of Theorem 3.1. Recall from (3.10) that the sequence of central binomial coefficients is an LP function. Further armed with (3.5) as well as Corollary 3.5 and Lemma 3.6, the claimed Lucas congruences for the sequences (a), (b), (c), (d), (f), $(\alpha),(\epsilon),(\gamma), s_{10}, s_{7}$ follow from (3.6). It remains to consider the sequences $(\mathrm{g}),(\delta),(\zeta)$ as well as $(\eta)$ and $s_{18}$.

Sequence $(\mathrm{g})$ can be written as

$$
A_{g}(n)=\sum_{k=0}^{n}(-1)^{k} 8^{n-k}\left(\begin{array}{l}
n \\
k
\end{array}\right) F(k)
$$

where $F(k)=\sum_{l=0}^{k}\left(\begin{array}{c}k \\ l\end{array}\right)^{3}$ are the Franel numbers (sequence (a)), which we already know to be an $\mathbf{L P}$ function. As a consequence of Fermat's little theorem, the sequence $a^{n}$ is an $\mathbf{L P}$ function for any integer $a$. Hence, Eq. (3.6) applies to show that $A_{g}(n)$ is an LP function.

In order to see that sequence $(\delta)$ satisfies the Lucas congruences as well, it suffices to observe that $L(n, k)=\left(\begin{array}{c}n+k \\ k\end{array}\right)$ is almost a DLP function, that is, it satisfies the congruences (3.4) but does not vanish for $k>n$. This is enough to conclude from Lemma 3.6 that

$$
L(n, k)=3^{n-3 k}\left(\begin{array}{c}
n \\
3 k
\end{array}\right)\left(\begin{array}{c}
n+k \\
k
\end{array}\right) \frac{(3 k) !}{k !^{3}}
$$


is a DLP function. Since this is the summand of sequence $(\delta)$, the desired Lucas congruences again follow from (3.6).

On the other hand, for sequence $(\zeta)$, we observe that

$$
L(n, k)=\sum_{j=0}^{n}\left(\begin{array}{l}
n \\
j
\end{array}\right)\left(\begin{array}{l}
k \\
j
\end{array}\right)\left(\begin{array}{c}
k+j \\
n
\end{array}\right)
$$

satisfies the congruences ( 3.4 ) by Lemma 3.3 because the summand is a TLP function by Lemma 3.4. Hence, $\left(\begin{array}{l}n \\ k\end{array}\right)^{2} L(n, k)$ is a DLP function. Writing sequence $(\zeta)$ as

$$
A_{\zeta}(n)=\sum_{k=0}^{n}\left(\begin{array}{l}
n \\
k
\end{array}\right)^{2} L(n, k)
$$

the claimed congruences once more follow from (3.6).

\section{Proofs for the two remaining sequences}

The proof of the Lucas congruences in the previous section does not readily extend to the sequences $(\eta)$ and $s_{18}$ from Table 2 , because, in contrast to the other cases, the known binomial sums for these sequences do not have the property that their summands satisfy the double Lucas property. Let us first note that the binomial sums for $s_{18}$ and sequence $(\eta)$, given in (2.3) and Table 2, can be simplified at the expense of working with binomial coefficients with negative entries. Namely, we have

$$
s_{18}(n)=\sum_{k=0}^{n}(-1)^{k}\left(\begin{array}{l}
n \\
k
\end{array}\right)\left(\begin{array}{c}
2 k \\
k
\end{array}\right)\left(\begin{array}{c}
2(n-k) \\
n-k
\end{array}\right)\left(\begin{array}{c}
2 n-3 k \\
n
\end{array}\right)
$$

and

$$
A_{\eta}(n)=\sum_{k=0}^{n}(-1)^{k}\left(\begin{array}{l}
n \\
k
\end{array}\right)^{3}\left(\begin{array}{c}
4 n-5 k \\
3 n
\end{array}\right),
$$

where, as usual, for any integer $m \geq 0$ and any number $x$, we define

$$
\left(\begin{array}{l}
x \\
m
\end{array}\right)=\frac{x(x-1) \cdots(x-m+1)}{m !} .
$$

For instance, the equivalence between (2.3) and (4.1) is a simple consequence of the fact that, for integers $n \geq 0$ and $l=n-k$,

$$
(-1)^{k}\left(\begin{array}{c}
2 n-3 k \\
n
\end{array}\right)=(-1)^{k+n}\left(\begin{array}{c}
-n+3 k-1 \\
n
\end{array}\right)=(-1)^{l}\left(\begin{array}{c}
2 n-3 l-1 \\
n
\end{array}\right) .
$$

For the first equality, we used that, for integers $b \geq 0$,

$$
\begin{aligned}
\left(\begin{array}{l}
a \\
b
\end{array}\right) & =\frac{a(a-1) \cdots(a-b+1)}{b !} \\
& =(-1)^{b} \frac{(-a)(-a+1) \cdots(-a+b-1)}{b !}=(-1)^{b}\left(\begin{array}{c}
-a+b-1 \\
b
\end{array}\right) .
\end{aligned}
$$

The following result generalizes the Lucas congruences for the sequence $s_{18}(n)$.

Theorem 4.1. Suppose that $B(n, k)$ is a DLP function with the property that $B(n, k)=$ $B(n, n-k)$. Then, the sequence

$$
A(n)=\sum_{k=0}^{n}(-1)^{k} B(n, k)\left(\begin{array}{c}
2 n-3 k \\
n
\end{array}\right)
$$

is an LP function, that is, A(n) satisfy the Lucas congruences (3.2). 
Proof. Let $p$ be a prime and let $n \geq 0$ be an integer. Write $n=n_{0}+n^{\prime} p$ and $k=k_{0}+k^{\prime} p$, where $0 \leq n_{0}<p$ and $0 \leq k_{0}<p$ and $n^{\prime}, k^{\prime}$ are nonnegative integers. We have to show that

$$
A(n) \equiv A\left(n_{0}\right) A\left(n^{\prime}\right) \quad(\bmod p)
$$

In the sequel, we denote

$$
C(n, k)=(-1)^{k} B(n, k)\left(\begin{array}{c}
2 n-3 k \\
n
\end{array}\right) .
$$

For $k_{0} \leq n_{0} / 3$, we have $2 n_{0}-3 k_{0} \geq n_{0} \geq 0$ and $2 n_{0}-3 k_{0} \leq 2 n_{0}<n_{0}+p$. Hence, by the usual argument, we have

$$
\begin{aligned}
\left(\begin{array}{c}
2 n-3 k \\
n
\end{array}\right) & \equiv\left[x^{n_{0}}\left(x^{p}\right)^{n^{\prime}}\right](1+x)^{2 n_{0}-3 k_{0}}\left(1+x^{p}\right)^{2 n^{\prime}-3 k^{\prime}} \quad(\bmod p) \\
& \equiv\left(\begin{array}{c}
2 n_{0}-3 k_{0} \\
n_{0}
\end{array}\right)\left(\begin{array}{c}
2 n^{\prime}-3 k^{\prime} \\
n^{\prime}
\end{array}\right) \quad(\bmod p) .
\end{aligned}
$$

Hence, we find that, when $k_{0} \leq n_{0} / 3$,

$$
C(n, k) \equiv C\left(n_{0}, k_{0}\right) C\left(n^{\prime}, k^{\prime}\right) \quad(\bmod p)
$$

For $n_{0} / 3<k_{0}<2 n_{0} / 3$, we have $n_{0}>2 n_{0}-3 k_{0}>0$. By the same argument as above, we find that

$$
\left(\begin{array}{c}
2 n-3 k \\
n
\end{array}\right) \equiv 0 \quad(\bmod p)
$$

and hence $C(n, k) \equiv C\left(n_{0}, k_{0}\right) \equiv 0$ modulo $p$.

Finally, consider the case $n_{0} \geq 1$ and $2 n_{0} / 3 \leq k_{0} \leq n_{0}$. In that case, $-p<-n_{0} \leq$ $2 n_{0}-3 k_{0} \leq 0$ or, equivalently, $0<2 n_{0}-3 k_{0}+p \leq p$. Hence, we have, modulo $p$,

$$
\begin{aligned}
\left(\begin{array}{c}
2 n-3 k \\
n
\end{array}\right) & \equiv\left[x^{n_{0}}\left(x^{p}\right)^{n^{\prime}}\right](1+x)^{2 n_{0}-3 k_{0}+p}\left(1+x^{p}\right)^{2 n^{\prime}-3 k^{\prime}-1} \\
& \equiv\left(\begin{array}{c}
2 n_{0}-3 k_{0}+p \\
n_{0}
\end{array}\right)\left(\begin{array}{c}
2 n^{\prime}-3 k^{\prime}-1 \\
n^{\prime}
\end{array}\right) \\
& \equiv\left(\begin{array}{c}
2 n_{0}-3 k_{0} \\
n_{0}
\end{array}\right)\left(\begin{array}{c}
2 n^{\prime}-3 k^{\prime}-1 \\
n^{\prime}
\end{array}\right)
\end{aligned}
$$

because, for any integers $A, B$ and $m$ such that $0 \leq m<p$,

$$
\begin{aligned}
\left(\begin{array}{c}
A+B p \\
m
\end{array}\right) & =\frac{1}{m !}(A+B p)(A+B p-1) \cdots(A+B p-m+1) \\
& \equiv \frac{1}{m !} A(A-1) \cdots(A-m+1)=\left(\begin{array}{c}
A \\
m
\end{array}\right) \quad(\bmod p)
\end{aligned}
$$

Set $l^{\prime}=n^{\prime}-k^{\prime}$. Applying (4.3) to the second binomial factor in (4.8), we find that

$$
\left(\begin{array}{c}
2 n-3 k \\
n
\end{array}\right) \equiv(-1)^{n^{\prime}}\left(\begin{array}{c}
2 n_{0}-3 k_{0} \\
n_{0}
\end{array}\right)\left(\begin{array}{c}
2 n^{\prime}-3 l^{\prime} \\
n^{\prime}
\end{array}\right) \quad(\bmod p)
$$

In combination with the assumed symmetry of $B(n, k)$, we therefore have that, when $n_{0} \geq 1$ and $2 n_{0} / 3 \leq k_{0} \leq n_{0}$,

$$
C(n, k) \equiv C\left(n_{0}, k_{0}\right) C\left(n^{\prime}, n^{\prime}-k^{\prime}\right) \quad(\bmod p) .
$$


We are now ready to combine all cases. First, suppose that $n_{0} \geq 1$. Noting that $k \leq n / 3$ implies $k^{\prime} \leq n^{\prime} / 3$, and using (4.6), (4.7) and (4.10), we conclude that, modulo $p$,

$$
\begin{aligned}
A(n) & =\sum_{k_{0}=0}^{p-1} \sum_{k^{\prime}=0}^{n^{\prime}} C(n, k) \equiv \sum_{k_{0}=0}^{n_{0}} \sum_{k^{\prime}=0}^{n^{\prime}} C(n, k) \\
& \equiv \sum_{k_{0}=0}^{\left\lfloor n_{0} / 3\right\rfloor} \sum_{k^{\prime}=0}^{n^{\prime}} C(n, k)+\sum_{k_{0}=\left\lceil 2 n_{0} / 3\right\rceil}^{n_{0}} \sum_{k^{\prime}=0}^{n^{\prime}} C(n, k) \\
& \equiv \sum_{k_{0}=0}^{\left\lfloor n_{0} / 3\right\rfloor} C\left(n_{0}, k_{0}\right) \sum_{k^{\prime}=0}^{n^{\prime}} C\left(n^{\prime}, k^{\prime}\right)+\sum_{k_{0}=\left\lceil 2 n_{0} / 3\right\rceil}^{n_{0}} C\left(n_{0}, k_{0}\right) \sum_{k^{\prime}=0}^{n^{\prime}} C\left(n^{\prime}, n^{\prime}-k^{\prime}\right) \\
& =\left[\sum_{k_{0}=0}^{\left\lfloor n_{0} / 3\right\rfloor} C\left(n_{0}, k_{0}\right)+\sum_{k_{0}=\left\lceil 2 n_{0} / 3\right\rceil}^{n_{0}} C\left(n_{0}, k_{0}\right)\right] \sum_{k^{\prime}=0}^{n^{\prime}} C\left(n^{\prime}, k^{\prime}\right) \\
& =A\left(n_{0}\right) A\left(n^{\prime}\right),
\end{aligned}
$$

which is what we wanted to prove. The case $n_{0}=0$ is simpler, and we only have to use (4.6) to again conclude that (4.5) holds.

Corollary 4.2. The sequence $s_{18}(n)$ satisfies the Lucas congruences (3.2).

Proof. Recall from the discussion in Section 3 that

$$
B(n, k)=\left(\begin{array}{l}
n \\
k
\end{array}\right)\left(\begin{array}{c}
2 k \\
k
\end{array}\right)\left(\begin{array}{c}
2(n-k) \\
n-k
\end{array}\right)
$$

is a DLP function. Obviously, $B(n, k)=B(n, n-k)$. Hence, Theorem 4.1 applies to show that $s_{18}(n)$, in the form (4.1) satisfies the Lucas congruences (3.2).

Next, we prove that the sequence $(\eta)$, which corresponds to the choice $a=3$ in Theorem 4.3, satisfies Lucas congruences as well.

Theorem 4.3. Let $a \in\{1,3\}$. Then, the sequence

$$
A(n)=\sum_{k=0}^{n}(-1)^{k}\left(\begin{array}{l}
n \\
k
\end{array}\right)^{a}\left(\begin{array}{c}
4 n-5 k \\
3 n
\end{array}\right)
$$

is an LP function, that is, A(n) satisfy the Lucas congruences (3.2).

Proof. Let $p$ be a prime and let $n \geq 0$ be an integer. As in the proof of Theorem 4.1, we write $n=n_{0}+n^{\prime} p$ and $k=k_{0}+k^{\prime} p$, where $0 \leq n_{0}<p$ and $0 \leq k_{0}<p$ and $n^{\prime}, k^{\prime}$ are nonnegative integers. Again, we have to show that

$$
A(n) \equiv A\left(n_{0}\right) A\left(n^{\prime}\right) \quad(\bmod p)
$$

Throughout the proof, let $d=\left\lfloor 3 n_{0} / p\right\rfloor$.

If $k_{0} \leq n_{0} / 5$, then $4 n_{0}-5 k_{0} \geq 3 n_{0} \geq 0$ and $4 n_{0}-5 k_{0} \leq 4 n_{0}<3 n_{0}+p$. Since $d=\left\lfloor 3 n_{0} / p\right\rfloor$, we thus have $0 \leq 3 n_{0}-d p<p$ and $0 \leq 4 n_{0}-5 k_{0}-d p<\left(3 n_{0}-d p\right)+p$. Therefore, modulo $p$, 


$$
\begin{aligned}
\left(\begin{array}{c}
4 n-5 k \\
3 n
\end{array}\right) & \equiv\left[x^{3 n_{0}-d p}\left(x^{p}\right)^{3 n^{\prime}+d}\right](1+x)^{4 n_{0}-5 k_{0}-d p}\left(1+x^{p}\right)^{4 n^{\prime}-5 k^{\prime}+d} \\
& \equiv\left(\begin{array}{c}
4 n_{0}-5 k_{0}-d p \\
3 n_{0}-d p
\end{array}\right)\left(\begin{array}{c}
4 n^{\prime}-5 k^{\prime}+d \\
3 n^{\prime}+d
\end{array}\right) \\
& \equiv\left(\begin{array}{c}
4 n_{0}-5 k_{0} \\
3 n_{0}
\end{array}\right)\left(\begin{array}{c}
4 n^{\prime}-5 k^{\prime}+d \\
3 n^{\prime}+d
\end{array}\right)
\end{aligned}
$$

where in the last step we used that, modulo $p$,

$$
\left(\begin{array}{c}
4 n_{0}-5 k_{0}-d p \\
3 n_{0}-d p
\end{array}\right)=\left(\begin{array}{c}
4 n_{0}-5 k_{0}-d p \\
n_{0}-5 k_{0}
\end{array}\right) \equiv\left(\begin{array}{c}
4 n_{0}-5 k_{0} \\
n_{0}-5 k_{0}
\end{array}\right)=\left(\begin{array}{c}
4 n_{0}-5 k_{0} \\
3 n_{0}
\end{array}\right)
$$

which follows from (4.9) because $0 \leq n_{0}-5 k_{0}<p$. In particular, we have

$$
\begin{aligned}
& \sum_{k_{0}=0}^{\left\lfloor n_{0} / 5\right\rfloor} \sum_{k^{\prime}=0}^{n^{\prime}}(-1)^{k}\left(\begin{array}{l}
n \\
k
\end{array}\right)^{a}\left(\begin{array}{c}
4 n-5 k \\
3 n
\end{array}\right) \\
\equiv & \sum_{k_{0}=0}^{\left\lfloor n_{0} / 5\right\rfloor}(-1)^{k_{0}}\left(\begin{array}{l}
n_{0} \\
k_{0}
\end{array}\right)^{a}\left(\begin{array}{c}
4 n_{0}-5 k_{0} \\
3 n_{0}
\end{array}\right) \sum_{k^{\prime}=0}^{n^{\prime}}(-1)^{k^{\prime}}\left(\begin{array}{c}
n^{\prime} \\
k^{\prime}
\end{array}\right)^{a}\left(\begin{array}{c}
4 n^{\prime}-5 k^{\prime}+d \\
3 n^{\prime}+d
\end{array}\right),
\end{aligned}
$$

and we observe that, for $d \in\{0,1\}$,

$$
A(n)=\sum_{k=0}^{n}(-1)^{k}\left(\begin{array}{l}
n \\
k
\end{array}\right)^{a}\left(\begin{array}{c}
4 n-5 k+d \\
3 n+d
\end{array}\right) .
$$

To see this, note that the the sum of the $k$-th and $(n-k)$-th term does not depend on the value of $d \in\{0,1\}$. Indeed, using (4.4), Pascal's relation and (4.4) again, we deduce that

$$
\begin{aligned}
& \left(\begin{array}{c}
4 n-5 k+1 \\
3 n+1
\end{array}\right)+(-1)^{n}\left(\begin{array}{c}
4 n-5(n-k)+1 \\
3 n+1
\end{array}\right) \\
= & \left(\begin{array}{c}
4 n-5 k+1 \\
3 n+1
\end{array}\right)-\left(\begin{array}{c}
4 n-5 k-1 \\
3 n+1
\end{array}\right) \\
= & {\left[\left(\begin{array}{c}
4 n-5 k+1 \\
3 n+1
\end{array}\right)-\left(\begin{array}{c}
4 n-5 k \\
3 n+1
\end{array}\right)\right]+\left[\left(\begin{array}{c}
4 n-5 k \\
3 n+1
\end{array}\right)-\left(\begin{array}{c}
4 n-5 k-1 \\
3 n+1
\end{array}\right)\right] } \\
= & \left(\begin{array}{c}
4 n-5 k \\
3 n
\end{array}\right)+\left(\begin{array}{c}
4 n-5 k-1 \\
3 n
\end{array}\right) \\
= & \left(\begin{array}{c}
4 n-5 k \\
3 n
\end{array}\right)+(-1)^{n}\left(\begin{array}{c}
4 n-5(n-k) \\
3 n
\end{array}\right) .
\end{aligned}
$$

Next, suppose that $n_{0} \geq 1$ and $4 n_{0} / 5 \leq k_{0} \leq n_{0}$. In that case, $-p<-n_{0} \leq 4 n_{0}-5 k_{0} \leq$ 0 or, equivalently, $0<4 n_{0}-5 k_{0}+p \leq p$. Hence, we have, modulo $p$,

$$
\begin{aligned}
\left(\begin{array}{c}
4 n-5 k \\
3 n
\end{array}\right) & \equiv\left[x^{3 n_{0}-d p}\left(x^{p}\right)^{3 n^{\prime}+d}\right](1+x)^{4 n_{0}-5 k_{0}+p}\left(1+x^{p}\right)^{4 n^{\prime}-5 k^{\prime}-1} \\
& \equiv\left(\begin{array}{c}
4 n_{0}-5 k_{0}+p \\
3 n_{0}-d p
\end{array}\right)\left(\begin{array}{c}
4 n^{\prime}-5 k^{\prime}-1 \\
3 n^{\prime}+d
\end{array}\right) .
\end{aligned}
$$


We rewrite the first binomial factor as follows, applying first (4.4) and then (4.9) twice, to find that, with $l_{0}=n_{0}-k_{0}$, modulo $p$,

$$
\begin{aligned}
\left(\begin{array}{c}
4 n_{0}-5 k_{0}+p \\
3 n_{0}-d p
\end{array}\right) & =(-1)^{n_{0}+d}\left(\begin{array}{c}
4 n_{0}-5 l_{0}-(d+1) p-1 \\
3 n_{0}-d p
\end{array}\right) \\
& \equiv(-1)^{n_{0}+d}\left(\begin{array}{c}
4 n_{0}-5 l_{0}-d p-1 \\
3 n_{0}-d p
\end{array}\right) \\
& =(-1)^{n_{0}+d}\left(\begin{array}{c}
4 n_{0}-5 l_{0}-d p-1 \\
n_{0}-5 l_{0}-1
\end{array}\right) \\
& \equiv(-1)^{n_{0}+d}\left(\begin{array}{c}
4 n_{0}-5 l_{0}-1 \\
n_{0}-5 l_{0}-1
\end{array}\right) \\
& =(-1)^{n_{0}+d}\left(\begin{array}{c}
4 n_{0}-5 l_{0}-1 \\
3 n_{0}
\end{array}\right) .
\end{aligned}
$$

Here, we proceeded under the assumption that $n_{0}-5 l_{0}>0$. It is straightforward to check that the final congruence also holds when $n_{0}=5 l_{0}$, because then the binomial coefficients vanish modulo $p$. We conclude that, when $n_{0} \geq 1$ and $4 n_{0} / 5 \leq k_{0} \leq n_{0}$,

$$
(-1)^{k}\left(\begin{array}{c}
4 n-5 k \\
3 n
\end{array}\right) \equiv(-1)^{l_{0}}\left(\begin{array}{c}
4 n_{0}-5 l_{0}-1 \\
3 n_{0}
\end{array}\right)(-1)^{k^{\prime}+d}\left(\begin{array}{c}
4 n^{\prime}-5 k^{\prime}-1 \\
3 n^{\prime}+d
\end{array}\right) \quad(\bmod p) .
$$

In particular, we have

$$
\begin{aligned}
& \sum_{k_{0}=\left\lceil 4 n_{0} / 5\right\rceil}^{n_{0}} \sum_{k^{\prime}=0}^{n^{\prime}}(-1)^{k}\left(\begin{array}{l}
n \\
k
\end{array}\right)^{a}\left(\begin{array}{c}
4 n-5 k \\
3 n
\end{array}\right) \\
\equiv & \sum_{k_{0}=\left\lceil 4 n_{0} / 5\right\rceil}^{n_{0}}(-1)^{l_{0}}\left(\begin{array}{l}
n_{0} \\
l_{0}
\end{array}\right)^{a}\left(\begin{array}{c}
4 n_{0}-5 l_{0}-1 \\
3 n_{0}
\end{array}\right) \sum_{k^{\prime}=0}^{n^{\prime}}(-1)^{k^{\prime}+d}\left(\begin{array}{l}
n^{\prime} \\
k^{\prime}
\end{array}\right)^{a}\left(\begin{array}{c}
4 n^{\prime}-5 k^{\prime}-1 \\
3 n^{\prime}+d
\end{array}\right) \\
= & \sum_{k_{0}=0}^{\left\lfloor n_{0} / 5\right\rfloor}(-1)^{k_{0}}\left(\begin{array}{l}
n_{0} \\
k_{0}
\end{array}\right)^{a}\left(\begin{array}{c}
4 n_{0}-5 k_{0}-1 \\
3 n_{0}
\end{array}\right) \sum_{k^{\prime}=0}^{n^{\prime}}(-1)^{k^{\prime}+d}\left(\begin{array}{c}
n^{\prime} \\
k^{\prime}
\end{array}\right)^{a}\left(\begin{array}{c}
4 n^{\prime}-5 k^{\prime}-1 \\
3 n^{\prime}+d
\end{array}\right),
\end{aligned}
$$

and we observe that, for integers $d \geq 0$,

$$
\sum_{k=0}^{n}(-1)^{k+d}\left(\begin{array}{l}
n \\
k
\end{array}\right)^{a}\left(\begin{array}{c}
4 n-5 k-1 \\
3 n+d
\end{array}\right)=\sum_{k=0}^{n}(-1)^{k}\left(\begin{array}{l}
n \\
k
\end{array}\right)^{a}\left(\begin{array}{c}
4 n-5 k+d \\
3 n+d
\end{array}\right)
$$

because, by (4.4),

$$
(-1)^{k}\left(\begin{array}{c}
4 n-5 k+d \\
3 n+d
\end{array}\right)=(-1)^{(n-k)+d}\left(\begin{array}{c}
4 n-5(n-k)-1 \\
3 n+d
\end{array}\right) .
$$

Therefore, we can combine (4.14) and (4.16) into

$$
\begin{gathered}
\sum_{\substack{k_{0}=0 \\
k_{0} \leq n_{0} / 5 \text { or } k_{0} \geq 4 n_{0} / 5}}^{n_{0}} \sum_{k^{\prime}=0}^{n^{\prime}}(-1)^{k}\left(\begin{array}{l}
n \\
k
\end{array}\right)^{a}\left(\begin{array}{c}
4 n-5 k \\
3 n
\end{array}\right) \\
\equiv A\left(n_{0}\right) \sum_{k^{\prime}=0}^{n^{\prime}}(-1)^{k^{\prime}}\left(\begin{array}{c}
n^{\prime} \\
k^{\prime}
\end{array}\right)^{a}\left(\begin{array}{c}
4 n^{\prime}-5 k^{\prime}+d \\
3 n^{\prime}+d
\end{array}\right)(\bmod p),
\end{gathered}
$$

which holds for all $0 \leq n_{0}<p$ (recall from the discussion at the beginning of this section that $A\left(n_{0}\right)$, like sequence $(\eta)$, can be represented as in Table 2$)$. 
On the other hand, suppose that $n_{0} / 5<k_{0}<4 n_{0} / 5$. Set $f=\left\lfloor\left(4 n_{0}-5 k_{0}\right) / p\right\rfloor$. Since $0<4 n_{0}-5 k_{0}<3 n_{0}<3 p$, we have $f \in\{0,1,2\}$. The usual arguments show that, modulo $p$,

$$
\begin{aligned}
\left(\begin{array}{c}
4 n-5 k \\
3 n
\end{array}\right) & \equiv\left[x^{3 n_{0}-d p}\left(x^{p}\right)^{3 n^{\prime}+d}\right](1+x)^{4 n_{0}-5 k_{0}-f p}\left(1+x^{p}\right)^{4 n^{\prime}-5 k^{\prime}+f} \\
& \equiv\left(\begin{array}{c}
4 n_{0}-5 k_{0}-f p \\
3 n_{0}-d p
\end{array}\right)\left(\begin{array}{c}
4 n^{\prime}-5 k^{\prime}+f \\
3 n^{\prime}+d
\end{array}\right) \\
& \equiv\left(\begin{array}{c}
4 n_{0}-5 k_{0} \\
3 n_{0}-d p
\end{array}\right)\left(\begin{array}{c}
4 n^{\prime}-5 k^{\prime}+f \\
3 n^{\prime}+d
\end{array}\right) .
\end{aligned}
$$

We are now in a position to begin piecing everything together. To do so, we consider individually the cases corresponding to the value of $d \in\{0,1,2\}$.

First, suppose $d=0$ or $d=1$. Congruence (4.17) coupled with (4.15) implies that

$$
\sum_{\substack{k_{0}=0 \\
k_{0} \leq n_{0} / 5 \text { or } k_{0} \geq 4 n_{0} / 5}}^{n_{0}} \sum_{k^{\prime}=0}^{n^{\prime}}(-1)^{k}\left(\begin{array}{l}
n \\
k
\end{array}\right)^{a}\left(\begin{array}{c}
4 n-5 k \\
3 n
\end{array}\right) \equiv A\left(n_{0}\right) A\left(n^{\prime}\right) \quad(\bmod p) .
$$

To conclude the desired congruence (4.12), it therefore only remains to show that

$$
\sum_{k_{0}=\left\lfloor n_{0} / 5\right\rfloor+1}^{\left\lceil 4 n_{0} / 5\right\rceil-1} \sum_{k^{\prime}=0}^{n^{\prime}}(-1)^{k}\left(\begin{array}{l}
n \\
k
\end{array}\right)^{a}\left(\begin{array}{c}
4 n-5 k \\
3 n
\end{array}\right) \equiv 0 \quad(\bmod p)
$$

This is easily seen in the case $d=0$, because then each term of this sum vanishes modulo $p$. Equivalently, for $d=0$, (4.18) vanishes whenever $n_{0} / 5<k_{0}<4 n_{0} / 5$ (because $\left.0 \leq 4 n_{0}-5 k_{0}-f p \leq 4 n_{0}-5 k_{0}<3 n_{0}\right)$. On the other hand, if $d=1$, we claim that the sum (4.19) vanishes modulo $p$ because the terms corresponding to $\left(k_{0}, k^{\prime}\right)$ and $\left(k_{0}, n^{\prime}-k^{\prime}\right)$ cancel each other. To see that, observe first that, for $d=1$, (4.18) vanishes whenever $n_{0} / 5<k_{0}<4 n_{0} / 5$ and $f=\left\lfloor\left(4 n_{0}-5 k_{0}\right) / p\right\rfloor \neq 0$ (because $0 \leq 4 n_{0}-5 k_{0}-f p \leq$ $4 n_{0}-5 k_{0}-p<3 n_{0}-p$ if $\left.f \in\{1,2\}\right)$. Therefore, for the term corresponding to $\left(k_{0}, k^{\prime}\right)$,

$$
(-1)^{k}\left(\begin{array}{c}
4 n-5 k \\
3 n
\end{array}\right) \equiv(-1)^{k_{0}}\left(\begin{array}{c}
4 n_{0}-5 k_{0} \\
3 n_{0}-p
\end{array}\right)(-1)^{k^{\prime}}\left(\begin{array}{c}
4 n^{\prime}-5 k^{\prime} \\
3 n^{\prime}+1
\end{array}\right) \quad(\bmod p)
$$

while, for the term corresponding to $\left(k_{0}, n^{\prime}-k^{\prime}\right)$ with $j=k_{0}+\left(n^{\prime}-k^{\prime}\right) p$,

$$
\begin{aligned}
& (-1)^{j}\left(\begin{array}{c}
4 n-5 j \\
3 n
\end{array}\right) \equiv(-1)^{k_{0}}\left(\begin{array}{c}
4 n_{0}-5 k_{0} \\
3 n_{0}-p
\end{array}\right)(-1)^{n^{\prime}-k^{\prime}}\left(\begin{array}{c}
4 n^{\prime}-5\left(n^{\prime}-k^{\prime}\right) \\
3 n^{\prime}+1
\end{array}\right) \\
& \equiv(-1)^{k_{0}}\left(\begin{array}{c}
4 n_{0}-5 k_{0} \\
3 n_{0}-p
\end{array}\right)(-1)^{k^{\prime}+1}\left(\begin{array}{c}
4 n^{\prime}-5 k^{\prime} \\
3 n^{\prime}+1
\end{array}\right) \\
& \equiv-(-1)^{k}\left(\begin{array}{c}
4 n-5 k \\
3 n
\end{array}\right) \quad(\bmod p) \text {, }
\end{aligned}
$$

where we applied (4.4) for the second congruence. It is now immediate to see that the sum (4.19) indeed vanishes modulo $p$ for $d=1$. 
It remains to prove the Lucas congruences (4.12) in the case $d=2$. Using (4.17), we have

$$
A(n) \equiv A\left(n_{0}\right) \sum_{k^{\prime}=0}^{n^{\prime}}(-1)^{k^{\prime}}\left(\begin{array}{l}
n^{\prime} \\
k^{\prime}
\end{array}\right)^{a}\left(\begin{array}{c}
4 n^{\prime}-5 k^{\prime}+2 \\
3 n^{\prime}+2
\end{array}\right)+M \quad(\bmod p)
$$

where

$$
M:=\sum_{k_{0}=\left\lfloor n_{0} / 5\right\rfloor+1}^{\left\lceil 4 n_{0} / 5\right\rceil-1} \sum_{k^{\prime}=0}^{n^{\prime}}(-1)^{k}\left(\begin{array}{l}
n \\
k
\end{array}\right)^{a}\left(\begin{array}{c}
4 n-5 k \\
3 n
\end{array}\right) .
$$

Combining this congruence with the identity

$$
A(n)=\sum_{k=0}^{n}(-1)^{k}\left(\begin{array}{l}
n \\
k
\end{array}\right)^{a}\left[\left(\begin{array}{c}
4 n-5 k+2 \\
3 n+2
\end{array}\right)-\left(\begin{array}{c}
4 n-5 k \\
3 n+2
\end{array}\right)\right]
$$

which can be deduced along the same lines as (4.15), we find that

$$
A(n) \equiv A\left(n_{0}\right) A\left(n^{\prime}\right)+A\left(n_{0}\right) \sum_{k^{\prime}=0}^{n^{\prime}}(-1)^{k^{\prime}}\left(\begin{array}{l}
n^{\prime} \\
k^{\prime}
\end{array}\right)^{a}\left(\begin{array}{c}
4 n^{\prime}-5 k^{\prime} \\
3 n^{\prime}+2
\end{array}\right)+M \quad(\bmod p)
$$

We have, by (4.18), modulo $p$,

$$
\begin{aligned}
M & \equiv \sum_{k_{0}=\left\lfloor n_{0} / 5\right\rfloor+1}^{\left\lceil 4 n_{0} / 5\right\rceil-1}(-1)^{k_{0}}\left(\begin{array}{l}
n_{0} \\
k_{0}
\end{array}\right)^{a}\left(\begin{array}{c}
4 n_{0}-5 k_{0} \\
3 n_{0}-2 p
\end{array}\right) \sum_{k^{\prime}=0}^{n^{\prime}}(-1)^{k^{\prime}}\left(\begin{array}{l}
n^{\prime} \\
k^{\prime}
\end{array}\right)^{a}\left(\begin{array}{c}
4 n^{\prime}-5 k^{\prime}+f \\
3 n^{\prime}+2
\end{array}\right) \\
& \equiv \sum_{k_{0}=\left\lfloor n_{0} / 5\right\rfloor+1}^{\left\lceil 4 n_{0} / 5\right\rceil-1}(-1)^{k_{0}}\left(\begin{array}{l}
n_{0} \\
k_{0}
\end{array}\right)^{a}\left(\begin{array}{c}
4 n_{0}-5 k_{0} \\
3 n_{0}-2 p
\end{array}\right) \sum_{k^{\prime}=0}^{n^{\prime}}(-1)^{k^{\prime}}\left(\begin{array}{c}
n^{\prime} \\
k^{\prime}
\end{array}\right)^{a}\left(\begin{array}{c}
4 n^{\prime}-5 k^{\prime} \\
3 n^{\prime}+2
\end{array}\right),
\end{aligned}
$$

where the last congruence is a consequence of the identity

$$
\sum_{k=0}^{n}(-1)^{k}\left(\begin{array}{l}
n \\
k
\end{array}\right)^{a}\left(\begin{array}{c}
4 n-5 k+1 \\
3 n+2
\end{array}\right)=\sum_{k=0}^{n}(-1)^{k}\left(\begin{array}{l}
n \\
k
\end{array}\right)^{a}\left(\begin{array}{c}
4 n-5 k \\
3 n+2
\end{array}\right)
$$

(which follows from (4.4) and replacing $k$ with $n-k$ ) and the fact that (4.18) vanishes for $n_{0} / 5<k_{0}<4 n_{0} / 5$ if $f=2$. Using this value of $M$ in (4.20), we find that the desired Lucas congruence (4.12) follows, if we can show that

$$
A\left(n_{0}\right)+\sum_{k_{0}=\left\lfloor n_{0} / 5\right\rfloor+1}^{\left\lceil 4 n_{0} / 5\right\rceil-1}(-1)^{k_{0}}\left(\begin{array}{l}
n_{0} \\
k_{0}
\end{array}\right)^{a}\left(\begin{array}{c}
4 n_{0}-5 k_{0} \\
3 n_{0}-2 p
\end{array}\right) \equiv 0 \quad(\bmod p) .
$$

Note that, if $k_{0} \leq n_{0} / 5$, then, by (4.9) and (4.13),

$$
\left(\begin{array}{c}
4 n_{0}-5 k_{0} \\
3 n_{0}-2 p
\end{array}\right) \equiv\left(\begin{array}{c}
4 n_{0}-5 k_{0}-2 p \\
3 n_{0}-2 p
\end{array}\right) \equiv\left(\begin{array}{c}
4 n_{0}-5 k_{0} \\
3 n_{0}
\end{array}\right) \quad(\bmod p) .
$$

A similar argument, combined with (4.4), shows that the congruence (4.22) also holds if $k_{0} \geq 4 n_{0} / 5$. We therefore find that (4.21) is equivalent to

$$
\sum_{k_{0}=0}^{n_{0}}(-1)^{k_{0}}\left(\begin{array}{l}
n_{0} \\
k_{0}
\end{array}\right)^{a}\left(\begin{array}{c}
4 n_{0}-5 k_{0} \\
3 n_{0}-2 p
\end{array}\right) \equiv 0 \quad(\bmod p) .
$$

The next lemma proves that this congruence indeed holds provided that $a \in\{1,3\}$. 
Lemma 4.4. Let $p$ be a prime, and $a \in\{1,2,3\}$. Then we have, for all $n$ such that $2 p / 3 \leq$ $n<p$

$$
\sum_{k=0}^{n}(-1)^{a k}\left(\begin{array}{l}
n \\
k
\end{array}\right)^{a}\left(\begin{array}{l}
4 n-5 k \\
3 n-2 p
\end{array}\right) \equiv 0 \quad(\bmod p) .
$$

Proof. To prove these congruences we employ N. Calkin's technique [9] for proving similar divisibility results for sums of powers of binomials (6.9). Denoting $r=p-n$, we have, by (4.4) and (4.9),

$$
\begin{aligned}
\sum_{k=0}^{n}(-1)^{a k}\left(\begin{array}{l}
n \\
k
\end{array}\right)^{a}\left(\begin{array}{c}
4 n-5 k \\
3 n-2 p
\end{array}\right) & =\sum_{k=0}^{p-r}(-1)^{a k}\left(\begin{array}{c}
p-r \\
k
\end{array}\right)^{a}\left(\begin{array}{c}
4 p-4 r-5 k \\
p-3 r
\end{array}\right) \\
& =\sum_{k=0}^{p-r}\left(\begin{array}{c}
k-p+r-1 \\
k
\end{array}\right)^{a}\left(\begin{array}{c}
4 p-4 r-5 k \\
p-3 r
\end{array}\right) \\
& \equiv \sum_{k=0}^{p-r}\left(\begin{array}{c}
k+r-1 \\
k
\end{array}\right)^{a}\left(\begin{array}{c}
4 p-4 r-5 k \\
p-3 r
\end{array}\right) \quad(\bmod p) .
\end{aligned}
$$

Clearly,

$$
\left(\begin{array}{c}
k+r-1 \\
k
\end{array}\right)=\frac{(k+1)(k+2) \cdots(k+r-1)}{(r-1) !}=\frac{(k+1)_{r-1}}{(r-1) !},
$$

where $(x)_{k}=x(x+1) \cdots(x+k-1)$ denotes the Pochhammer symbol (in particular, $\left.(x)_{0}=1\right)$. Likewise,

$$
\left(\begin{array}{c}
4 p-4 r-5 k \\
p-3 r
\end{array}\right)=\frac{(3 p-r-5 k+1)_{p-3 r}}{(p-3 r) !}
$$

Since $(r-1)$ ! and $(p-3 r)$ ! are not divisible by $p$, we have to show that

$$
\sum_{k=0}^{p-r}(k+1)_{r-1}^{a}(3 p-r-5 k+1)_{p-3 r} \equiv 0 \quad(\bmod p) .
$$

Since the polynomials $(x)_{k},(x)_{k-1}, \ldots,(x)_{0}$ form an integer basis for the space of all polynomials with integer coefficients and degree at most $k$, there exist integers $c_{0}, c_{1}, \ldots, c_{N}$ with $N=(a-1)(r-1)+p-3 r$ so that

$$
(k+1)_{r-1}^{a-1}(3 p-r-5 k+1)_{p-3 r}=\sum_{j=0}^{N} c_{j}(k+r)_{j} .
$$

Then the left-hand side of (4.24) becomes

$$
\begin{aligned}
\sum_{k=0}^{p-r}(k+1)_{r-1} \sum_{j=0}^{N} c_{j}(k+r)_{j} & =\sum_{j=0}^{N} c_{j} \sum_{k=0}^{p-r}(k+1)_{r-1}(k+r)_{j} \\
& =\sum_{j=0}^{N} c_{j} \sum_{k=0}^{p-r}(k+1)_{r+j-1} \\
& =\sum_{j=0}^{N} c_{j} \frac{(p-r+1)_{r+j}}{r+j},
\end{aligned}
$$

where we used

$$
(x)_{k}-(x-1)_{k}=k(x)_{k-1}
$$


to evaluate

$$
\sum_{k=0}^{p-r}(k+1)_{r+j-1}=\sum_{k=0}^{p-r} \frac{(k+1)_{r+j}-(k)_{r+j}}{r+j}=\frac{(p-r+1)_{r+j}}{r+j} .
$$

The desired congruence therefore follows if we can show that

$$
\frac{(p-r+1)_{r+j}}{r+j} \equiv 0 \quad(\bmod p)
$$

for all $j=0,1, \ldots, N$. Since $r>0$ and $j \geq 0$, the numerator $(p-r+1)_{r+j}$ is always divisible by $p$. The congruences (4.26) thus follow if $r+j<p$ for all $j$, or, equivalently, $r+N<p$. Since

$$
r+N=(a-1)(r-1)+p-2 r
$$

we have $r+N<p$ if and only if

$$
(a-1)(r-1)<2 r .
$$

Clearly, this inequality holds for all $r \geq 1$ if and only if $a \leq 3$.

Remark 4.5. Numerical evidence suggests that the values $a \in\{1,3\}$ in Theorem 4.3 are the only choices for which the sequence (4.11) satisfies Lucas congruences. In light of Lemma 4.4, it is natural to ask if there are additional values of $a$ and $\varepsilon$, for which the sequence

$$
\sum_{k=0}^{n}(-1)^{\varepsilon k}\left(\begin{array}{l}
n \\
k
\end{array}\right)^{a}\left(\begin{array}{c}
4 n-5 k \\
3 n
\end{array}\right)
$$

satisfies Lucas congruences. Empirically, this does not appear to be the case. In particular, for $a=2$ this sequence does not satisfy Lucas congruences for either $\varepsilon=0$ or $\varepsilon=1$.

\section{Periodicity of residues}

The Apéry numbers satisfy

$$
A(n) \equiv(-1)^{n} \quad(\bmod 3) \text {, }
$$

and so are periodic modulo 3. As in the case of the congruences (1.4), which show that the Apéry numbers are also periodic modulo 8, the congruences (5.1) were first conjectured in [11] and then proven in [16]. We say that a sequence $C(n)$ is eventually periodic if there exists an integer $M>0$ such that $C(n+M)=C(n)$ for all sufficiently large $n$. An initial numerical search suggests that each sporadic Apéry-like sequence listed in Tables 1 and 2 can only be eventually periodic modulo a prime $p$ if $p \leq 5$. As an application of Theorem 3.1, we prove this claim next.

Corollary 5.1. None of the sequences from Tables 1 and 2 is eventually periodic modulo p for any prime $p>5$.

Proof. Gessel [16] shows that, if a sequence $C(n)$ satisfies the Lucas congruences (3.2) modulo $p$ and is eventually periodic modulo $p$, then $C(n) \equiv C(1)^{n}$ modulo $p$ for all $n=$ $0,1, \ldots, p-1$. 
For instance, let $C(n)$ be the Almkvist-Zudilin sequence $(\delta)$. Then, $C(1)=3, C(2)=9$ and $C(3)=3$. Suppose $C(n)$ was eventually periodic modulo $p$. Then $p$ has to divide $C(3)-C(1)^{3}=-24$, which implies that $p \in\{2,3\}$.

In Table 3 we list, for each sequence, the primes dividing both $C(2)-C(1)^{2}$ and $C(3)-$ $C(1)^{3}$. The fact, that all these primes are at most 5 , proves our claim.

As another simple consequence of Theorem 3.1, we observe that the Apéry-like sequences are in fact eventually periodic modulo each of the primes listed in Table 3.

Corollary 5.2. Let $C(n)$ be any sequence from Tables 1 and 2 .

- $C(n) \equiv C(1)(\bmod 2)$ for all $n \geq 1$.

- $C(n) \equiv C(1)(\bmod 3)$ for all $n \geq 1$ if $C(n)$ is one of $(c),(f),(g),(\delta),(\alpha),(\epsilon),(\zeta), s_{18}$, and $C(n) \equiv(-1)^{n}(\bmod 3)$ for all $n \geq 0$ if $C(n)$ is $(a)$ or $(\gamma)$.

- $C(n) \equiv 3^{n}(\bmod 5)$ for all $n \geq 0$ if $C(n)$ is $(b)$, and $C(n) \equiv 0(\bmod 5)$ for all $n \geq 1$ if $C(n)$ is $(\eta)$.

Proof. One can check that Table 3 does not change if we include only those primes $p$ such that $C(n)-C(1)^{n}$ is divisible by $p$ for all $n \in\{0,1,2,3,4\}$. For $n=0$, this is trivial since $C(0)=1$. Therefore, in each of the cases considered here, we have

$$
C(n) \equiv C(1)^{n} \quad(\bmod p)
$$

for all $n \in\{0,1, \ldots, p-1\}$. For any $n \geq 0$, let $n=n_{0}+n_{1} p+\cdots+n_{r} p^{r}$ be the $p$-adic expansion of $n$. Then, by Theorem 3.1, we have

$$
\begin{aligned}
C(n) & \equiv C\left(n_{0}\right) C\left(n_{1}\right) \cdots C\left(n_{r}\right) \quad(\bmod p) \\
& \equiv C(1)^{n_{0}+n_{1}+\cdots+n_{r}} \quad(\bmod p) \\
& \equiv C(1)^{n} \quad(\bmod p) .
\end{aligned}
$$

For the final congruence we used Fermat's little theorem. All claimed congruences then follow from the specific initial values of $C(n)$ modulo $p$.

More interestingly, the congruences (1.4) show that the Apéry numbers (sequence $(\gamma)$ ) are periodic modulo 8 . We offer the following corresponding result for the AlmkvistZudilin sequence $(\delta)$.

Theorem 5.3. The Almkvist-Zudilin numbers

$$
Z(n)=\sum_{k=0}^{n}(-1)^{k} 3^{n-3 k}\left(\begin{array}{c}
n \\
3 k
\end{array}\right)\left(\begin{array}{c}
n+k \\
n
\end{array}\right) \frac{(3 k) !}{k !^{3}}
$$

Table 3 The primes dividing both $C(2)-C(1)^{2}$ and $C(3)-C(1)^{3}$, for each sequence $C(n)$ from Tables 1 and 2

\begin{tabular}{lllllllllllllll}
\hline (a) & $(\mathrm{b})$ & $(\mathrm{c})$ & $(\mathrm{d})$ & $(\mathrm{f})$ & $(\mathrm{g})$ & $(\delta)$ & $(\eta)$ & $(\alpha)$ & $(\epsilon)$ & $(\zeta)$ & $(\gamma)$ & $\left(s_{7}\right)$ & $\left(s_{10}\right)$ & $\left(s_{18}\right)$ \\
\hline 2,3 & 2,5 & 2,3 & 2 & 2,3 & 2,3 & 2,3 & 2,5 & 2,3 & 2,3 & 2,3 & 2,3 & 2 & 2 & 2,3 \\
\hline
\end{tabular}


satisfy the congruences

$$
Z(n) \equiv\left\{\begin{array}{l}
1, \text { if } n \text { is even, } \\
3, \text { if } n \text { is odd }
\end{array} \quad(\bmod 8)\right.
$$

Proof. It is shown in [28] that the numbers $(-1)^{n} Z(n)$ are the diagonal Taylor coefficients of the multivariate rational function

$$
F\left(x_{1}, x_{2}, x_{3}, x_{4}\right)=\frac{1}{1-\left(x_{1}+x_{2}+x_{3}+x_{4}\right)+27 x_{1} x_{2} x_{3} x_{4}} .
$$

That is, if

$$
F\left(x_{1}, x_{2}, x_{3}, x_{4}\right)=\sum_{n_{1}=0}^{\infty} \sum_{n_{2}=0}^{\infty} \sum_{n_{3}=0}^{\infty} \sum_{n_{4}=0}^{\infty} C\left(n_{1}, n_{2}, n_{3}, n_{4}\right) x_{1}^{n_{1}} x_{2}^{n_{2}} x_{3}^{n_{3}} x_{4}^{n_{4}}
$$

is the Taylor expansion of the rational function $F$, then $Z(n)=(-1)^{n} C(n, n, n, n)$.

Given such a rational function as well as a prime power $p^{r}$, Rowland and Yassawi [25] give an explicit algorithm for computing a finite state automaton, which produces the values of the diagonal coefficients modulo $p^{r}$. In the present case, this finite state automaton for the values $(-1)^{n} Z(n)$ modulo 8 turns out to be the same automaton as the one for the Apéry numbers modulo 8. Hence, the congruences (5.2) follow from the congruences (1.4). We refer to [25] for details on finite state automata and the algorithm to construct them from a multivariate rational generating function. We also remark that, due to the complexity of the algorithm, $p^{r}$ should be reasonably small in practice (for instance, the implementation accompanying [25] takes several minutes to compute the finite state automaton for the coefficients of (5.3) modulo $2^{5}$, and did not finish in reasonable time modulo $2^{6}$ ).

Empirically, Theorem 5.3 is the only other interesting set of congruences, apart from the congruences (1.4), which demonstrates that an Apéry-like sequence is periodic modulo a prime power. More precisely, numerical evidence suggests that none of the sequences in Tables 1 and 2 is eventually periodic modulo $p^{r}$, for some $r>1$, unless $p=2$. Moreover, the only other instances modulo a power of 2 appear to be the following, less interesting, ones: sequences $(\mathrm{d})$ and $(\alpha)$ are eventually periodic modulo 4 because all their terms, except the first, are divisible by 4 ; likewise, sequences $(\varepsilon)$ and $s_{7}$ are eventually periodic modulo 8 because all their terms, except the first, are divisible by 8 . We do not attempt to prove these claims here. We remark, however, that these claims can be established by the approach used in the proof of Theorem 5.3, provided that one is able to determine a computationally accessible analog of (5.3) for the sequence at hand.

\section{Primes not dividing Apéry-like numbers}

Using the Lucas congruences proved in Theorem 3.1, it is straightforward to verify whether or not a given prime divides some Apéry-like number.

Example 6.1. The values of Apéry numbers $A(0), A(1), \ldots, A(6)$ modulo 7 are $1,5,3,3,3,5,1$. Since 7 does not divide $A(0), A(1), \ldots, A(6)$, it follows from the Lucas congruences (3.2) that 7 does not divide any Apéry number. 
Arguing as in Example 6.1, one finds that the primes 2, 3, 7, 13, 23, 29, 43, 47, . do not divide any Apéry number $A(n)$. E. Rowland and R. Yassawi [25] pose the question whether there are infinitely many such primes. Table 4 records, for each sporadic Apéry-like sequence, the primes below 100 which do not divide any of its terms, and the last column gives the proportion of primes below $10^{4}$ with this property. Each Apéry-like sequence is specified by its label from [3], which is also used in Tables 1 and 2 . The alert reader will notice that Cooper's sporadic sequences (the ones with $d \neq 0$ in Table 2) are missing from Table 4. That is because these sequences turn out to be divisible by all primes. A more precise result for these sequences is proved at the end of this section.

Example 6.1 shows that the first 7 values of the Apéry numbers modulo 7 are palindromic. Our next result, which was noticed by E. Rowland, shows that this is true for all primes.

Lemma 6.2. For any prime $p$, and integers $n$ such that $0 \leq n<p$, the Apéry numbers $A(n)$ satisfy the congruence

$$
A(n) \equiv A(p-1-n) \quad(\bmod p)
$$

Proof. For $n$ such that $0 \leq n<p$, we employ (4.4) and (4.9) to arrive at

$$
\begin{aligned}
A(p-1-n) & =\sum_{k=0}^{p-1}\left(\begin{array}{c}
p-1-n \\
k
\end{array}\right)^{2}\left(\begin{array}{c}
p-1-n+k \\
k
\end{array}\right)^{2} \\
& \equiv \sum_{k=0}^{p-1}\left(\begin{array}{c}
n+k \\
k
\end{array}\right)^{2}\left(\begin{array}{l}
n \\
k
\end{array}\right)^{2}=A(n) \quad(\bmod p)
\end{aligned}
$$

as claimed.

Theorem 3.1 and Lemma 6.2 , considered together, suggest that $e^{-1 / 2} \approx 60.65 \%$ of the primes do not divide any Apéry number. Indeed, let us make the empirical assumption

\begin{tabular}{|c|c|c|}
\hline (a) & $3,11,17,19,43,83,89,97$ & 0.2994 \\
\hline (b) & $2,5,13,17,29,37,41,61,73,89$ & 0.2897 \\
\hline (c) & $2,7,13,37,61,73$ & 0.2962 \\
\hline (d) & $3,11,17,19,43,59,73,83,89$ & 0.2815 \\
\hline$(f)$ & $2,5,13,17,29,37,41,61,73,97$ & 0.2994 \\
\hline (g) & $5,11,29,31,59,79$ & 0.2929 \\
\hline$(\delta)$ & $2,5,7,11,13,19,29,41,47,61,67,71,73,89,97$ & 0.6192 \\
\hline$(\eta)$ & $2,3,17,19,23,31,47,53,61$ & 0.2897 \\
\hline$(\alpha)$ & $3,5,13,17,29,31,37,41,43,47,53,59,61,67,71,83,89$ & 0.5989 \\
\hline$(\epsilon)$ & $3,7,13,19,23,29,31,37,43,47,61,67,73,83,89$ & 0.6037 \\
\hline$(\zeta)$ & $2,5,7,13,17,19,29,37,43,47,59,61,67,71,83,89$ & 0.6046 \\
\hline$(\gamma)$ & $2,3,7,13,23,29,43,47,53,67,71,79,83,89$ & 0.6168 \\
\hline
\end{tabular}
that the values $A(n)$ modulo $p$, for $n=0,1, \ldots,(p-1) / 2$, are independent and uniformly

Table 4 Primes not dividing Apéry-like numbers

The primes below 100 not dividing Apéry-like numbers (sequence indicated in first column using the labels from [3]) as well as the proportion of primes (in the last column) below 10,000 not dividing any term 
random. Since one of the values $A(n)$ is congruent to 0 modulo $p$ with probability $1 / p$, it follows that the probability that $p$ does not divide any of the $(p+1) / 2$ first values is

$$
\left(1-\frac{1}{p}\right)^{(p+1) / 2} \text {. }
$$

By the Lucas congruences, shown in Theorem 3.1, and Lemma 6.2, $p$ does not divide any of the $(p+1) / 2$ first values if and only if $p$ does not divide any Apéry number. In the limit $p \rightarrow \infty$, the proportion (6.2) becomes $e^{-1 / 2}$. Observe that this empirical prediction matches the numerical data in Table 4 rather well. We therefore arrive at the following conjecture.

Conjecture 6.3. The proportion of primes not dividing any Apéry number $A(n)$ is $e^{-1 / 2}$.

While Lemma 6.2 does not hold for the other Apéry-like numbers $C(n)$ from Tables 1 and 2, we make the weaker observation that if a prime $p>5$ divides $C(n)$, where $0 \leq$ $n<p$, then $p$ also divides $C(p-1-n)$. We expect that this empirical observation can be proven in the spirit of the proof of Lemma 6.2, but do not pursue this theme further. We only note that it allows us to extend the heuristic leading to Conjecture 6.3 to the Apérylike sequences $(\delta),(\alpha),(\epsilon),(\zeta)$ from Table 2. In other words, we conjecture that, for each of these sequences, the proportion of primes not dividing any of the terms is again $e^{-1 / 2}$. Figure 1 visualizes some numerical evidence for this conjecture. On the other hand, for sequence $(\eta)$ as well as the sequences from Table 1, the proportion of primes not dividing any of their terms appears to be about half of that, that is $e^{-1 / 2} / 2 \approx 30.33 \%$.

To explain this extra factor of $1 / 2$, we note that, for the Apéry-like numbers

$$
A_{b}(n)=\sum_{k}\left(\begin{array}{l}
n \\
k
\end{array}\right)^{2}\left(\begin{array}{c}
n+k \\
n
\end{array}\right),
$$

Stienstra and Beukers [27] proved that, modulo $p$,

$$
A_{b}\left(\frac{p-1}{2}\right) \equiv \begin{cases}4 a^{2}-2 p, & \text { if } p=a^{2}+b^{2}, a \text { odd } \\ 0, & \text { if } p \equiv 3 \quad(\bmod 4)\end{cases}
$$

(and conjectured that the congruence should hold modulo $p^{2}$, which was later proved by Ahlgren [1]; see also [2]). In particular, congruence (6.4) makes it explicit that every

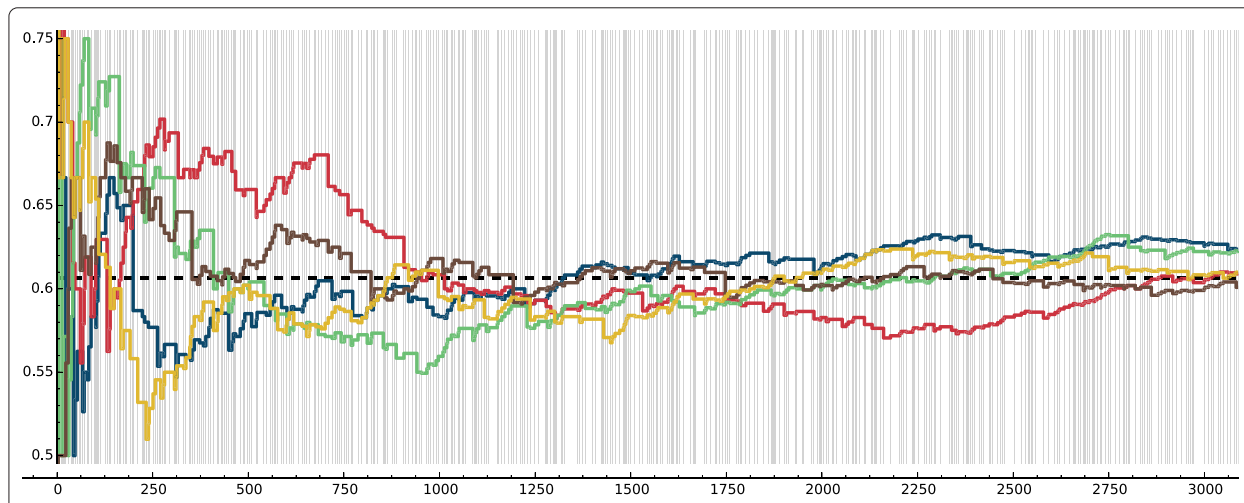

Fig. 1 Proportion of primes (up to 3000) not dividing the sequences $(\delta),(\alpha),(\epsilon),(\zeta),(\gamma)$, with the dotted line indicating $e^{-1 / 2}$. The Apéry sequence is plotted in blue. (We thank Arian Daneshvar for producing this plot.) 
prime $p \equiv 3(\bmod 4)$ divides a term of this Apéry-like sequence. Note that, by a classical congruence of Gauss, the congruences (6.4) are equivalent, modulo $p$, to the congruences

$$
A_{b}\left(\left\lfloor\frac{p}{2}\right\rfloor\right) \equiv \begin{cases}\left(\begin{array}{l}
\lfloor p / 2\rfloor \\
\lfloor p / 4\rfloor
\end{array}\right)^{2}, & \text { if } p \equiv 1 \quad(\bmod 4) \\
0, & \text { otherwise }\end{cases}
$$

which are valid for any prime $p \neq 2$. The more general result in [27] also includes the cases $A_{a}$ and $A_{c}$. Similar divisibility results appear to hold for the other Apéry-like numbers from Table 1, and it would be interesting to make these explicit.

On the other hand, the extra factor of $1 / 2$ in case of sequence $(\eta)$ is explained by the following congruences, which resemble (6.5) remarkably well.

Theorem 6.4. For any prime $p \neq 3$, we have that, modulo $p$,

$$
A_{\eta}\left(\left\lfloor\frac{p}{3}\right\rfloor\right) \equiv \begin{cases}(-1)^{\lfloor p / 5\rfloor}(\underset{\lfloor p / 15\rfloor}{\lfloor p / 3\rfloor})^{3}, & \text { if } p \equiv 1,2,4,8 \quad(\bmod 15), \\ 0, & \text { otherwise }\end{cases}
$$

Proof. Suppose that $p \equiv 2(\bmod 3)$, and write $p=3 n+2$. The congruence (6.6) can be checked directly for $p=2$ and $p=5$, and so we may assume $p>5$ in the sequel. Applying (4.23) to the definition of sequence $(\eta)$ in Table 2, we have

$$
\begin{aligned}
A_{\eta}(n) & =\sum_{k=0}^{\lfloor n / 5\rfloor}(-1)^{k}\left(\begin{array}{l}
n \\
k
\end{array}\right)^{3}\left(\left(\begin{array}{c}
4 n-5 k-1 \\
3 n
\end{array}\right)+\left(\begin{array}{c}
4 n-5 k \\
3 n
\end{array}\right)\right) \\
& =\sum_{k=0}^{\lfloor n / 5\rfloor}(-1)^{k}\left(\begin{array}{l}
n \\
k
\end{array}\right)^{3}\left(\frac{(n-5 k)_{3 n}}{(3 n) !}+\frac{(n-5 k+1)_{3 n}}{(3 n) !}\right) .
\end{aligned}
$$

Since $3 n=p-2$ and $0 \leq k \leq n / 5$, the term

$$
\frac{(n-5 k)_{3 n}}{(3 n) !}
$$

is always divisible by $p$, unless $n-5 k \in\{1,2\}$ (because, otherwise, one of the $p-2$ factors of $(n-5 k)_{3 n}$ is divisible by $p$, while ( $\left.3 n\right)$ ! is not). Note that $n-5 k=1$ and $n-5 k=2$ are equivalent to $k=(p-5) / 15$ and $k=(p-8) / 15$, respectively. However, $(p-5) / 15$ cannot be an integer (since $p \neq 5$ ). We thus find that (6.8) vanishes modulo $p$ unless $p \equiv 8$ $(\bmod 15)$ and $k=\lfloor p / 15\rfloor$, in which case (6.8) is congruent to -1 modulo $p$. Combined with the analogous discussion for the corresponding second term in (6.7), we conclude that

$$
\frac{(n-5 k)_{3 n}}{(3 n) !}+\frac{(n-5 k+1)_{3 n}}{(3 n) !} \equiv \begin{cases}1, & \text { if } k=\lfloor p / 15\rfloor \text { and } p \equiv 2 \quad(\bmod 15) \\ -1, & \text { if } k=\lfloor p / 15\rfloor \text { and } p \equiv 8 \\ 0, & \text { otherwise }\end{cases}
$$

Applying this to the sum (6.7) and combining the signs properly, we arrive at the congruences $(6.6)$ when $p \equiv 2(\bmod 3)$.

The case $p \equiv 1(\bmod 3)$ is similar and a little bit simpler.

In summary, we conjecture that the proportion of primes not dividing any term of the Apéry-like sequences in Tables 1 and 2 is as follows. 


\section{Conjecture 6.5.}

- Let $C(n)$ be one of the sequences of Table 1 or sequence $(\eta)$. Then the proportion of primes not dividing any $C(n)$ is $\frac{1}{2} e^{-1 / 2}$.

- Let $C(n)$ be one of the sequences $(\delta),(\alpha),(\epsilon),(\zeta),(\gamma)$ from Table 2 . Then the proportion of primes not dividing any $C(n)$ is $e^{-1 / 2}$.

In stark contrast, Cooper's sporadic sequences $s_{7}, s_{10}, s_{18}$ from Table 2 are divisible by all primes. Indeed, let $C(n)$ denote any of these three sequences. Then,

$$
C(p-1) \equiv 0 \quad(\bmod p)
$$

for all primes $p$. In fact, we can prove much more. For any given prime $p$, the last quarter (or third) of the first $p$ terms of these sequences are divisible by $p$. In the case of sequence $s_{10}$, the sum of fourth powers of binomial coefficients, this is proved by N. Calkin [9]. Indeed, among other divisibility results on sums of powers of binomials, Calkin shows that, for all integers $a \geq 0$, the sums

$$
\sum_{k=0}^{n}\left(\begin{array}{l}
n \\
k
\end{array}\right)^{2 a}
$$

are divisible by all primes $p$ in the range

$$
n<p<n+1+\frac{n}{2 a-1} \text {. }
$$

In particular, in the case $a=2$, we conclude that $s_{10}(n)$ is divisible by all primes $p$ that satisfy $n<p<\frac{4 n}{3}+1$. Equivalently, we have

$$
s_{10}(p-j) \equiv 0 \quad(\bmod p)
$$

whenever $1 \leq j \leq(p+2) / 4$. Our final result proves the same phenomenon for Cooper's sporadic sequences $s_{7}, s_{18}$. We note that in each case, empirically, the bounds on $j$ cannot be improved (with the expection of the case $p=3$ for $s_{18}$; see Remark 6.7).

Theorem 6.6. For any prime $p$, we have

$$
s_{7}(p-j) \equiv 0 \quad(\bmod p)
$$

whenever $1 \leq j \leq(p+1) / 3$, and

$$
s_{18}(p-j) \equiv 0 \quad(\bmod p)
$$

whenever $1 \leq j \leq(p+2) / 4$.

Proof. For the sequence $s_{7}$, we want to show

$$
\sum_{k=0}^{p-j}\left(\begin{array}{c}
p-j \\
k
\end{array}\right)^{2}\left(\begin{array}{c}
p-j+k \\
k
\end{array}\right)\left(\begin{array}{c}
2 k \\
p-j
\end{array}\right) \equiv 0 \quad(\bmod p),
$$

for $1 \leq j \leq(p+1) / 3$. Note that for $2 k<p-j$ or $k>p-j$ the summand is already zero. Therefore, we assume that $p-j \geq k \geq(p-j) / 2$. We write the summand as

$$
\left(\begin{array}{c}
p-j \\
k
\end{array}\right)^{2}\left(\begin{array}{c}
p-j+k \\
k
\end{array}\right)\left(\begin{array}{c}
2 k \\
p-j
\end{array}\right)=\frac{(p-j+k) !(2 k) !}{k !^{3}(p-j-k) !^{2}(2 k-p+j) !},
$$


and observe that the denominator is not divisible by $p$ if $j \geq 1$. On the other hand, the factorial $(p-j+k)$ ! in the numerator is divisible by $p$ since

$$
p-j+k \geq p-j+\left\lceil\frac{p-j}{2}\right\rceil \geq p,
$$

where we used $j \leq(p+1) / 3$ to verify the final inequality. Thus, for $1 \leq j \leq(p+1) / 3$, the congruences $s_{7}(p-j) \equiv 0$ hold modulo $p$, as claimed.

We proceed similarly for $s_{18}(p-j)$, which is given by

$$
\sum_{k=0}^{\lfloor(p-j) / 3\rfloor}(-1)^{k}\left(\begin{array}{c}
p-j \\
k
\end{array}\right)\left(\begin{array}{c}
2 k \\
k
\end{array}\right)\left(\begin{array}{c}
2(p-j-k) \\
p-j-k
\end{array}\right)\left\{\left(\begin{array}{c}
2(p-j)-3 k-1 \\
p-j
\end{array}\right)+\left(\begin{array}{c}
2(p-j)-3 k \\
p-j
\end{array}\right)\right\},
$$

and, using (4.23), write the summand as

$$
\frac{(-1)^{k}(2 k) !(2(p-j-k)) !}{k !^{3}(p-j-k) !^{!}}(p-j-3 k+1)_{p-j-1}(3 p-3 j-6 k) .
$$

None of the terms in the denominator is divisible by $p$ since $j \geq 1$. On the other hand, $(2(p-j-k))$ ! in the numerator is divisible by $p$ since

$$
2(p-j-k) \geq 2\left(p-j-\left\lfloor\frac{p-j}{3}\right\rfloor\right) \geq p,
$$

where we used $j \leq(p+2) / 4$ for the final inequality. Therefore, for $1 \leq j \leq(p+2) / 4$, each of the terms in the sum for $s_{18}(p-j)$ is a multiple of $p$, and we obtain the desired congruences.

Remark 6.7. Employing (6.10), we observe that $s_{18}(n) \equiv 0(\bmod 3)$ for $n \geq 1$, which reaffirms Corollary 5.2 for this sequence.

Finally, as noted in [12], each of the sequences in Table 1 times $\left(\begin{array}{c}2 n \\ n\end{array}\right)$ is an integer solution of (2.2) with $d \neq 0$. Observe that $\left(\begin{array}{c}2 n \\ n\end{array}\right)$ is divisible by a prime $p$ for all $n$ such that $n<p \leq$ $2 n$. This results in a (weaker) analog of Theorem 6.6 for these Apéry-like sequences, and implies, in particular, that these sequences are again divisible by all prime numbers.

\section{Conclusion and open questions}

In Sections 3 and 4, we showed that all sporadic solutions of (2.1) and (2.2), given in Tables 1 and 2, uniformly satisfy Lucas congruences. However, for two of these sequences, especially sequence $(\eta)$, we had to resort to a rather technical analysis. We therefore wonder if there is a representation of these sequences from which the Lucas congruences can be deduced more naturally, based on, for instance the approaches of [26] and [20], or [25]. More generally, it would be desirable to have a uniform approach to these congruences, possibly directly from the shape of the defining recurrences and associated differential equations. In another direction, it would be interesting to show that, as numerical evidence suggests, all of the Apéry-like sequences in fact satisfy the Dwork congruences (1.3).

The congruences (1.4) show that the Apéry numbers are periodic modulo 8 , alternating between the values 1 and 5 . As a consequence, the other residue classes $0,2,3,4,6,7$ modulo 8 are never attained. On the other hand, the observations in Section 6 show that certain primes do not divide any Apéry number. This can be rephrased as saying that the residue class 0 is not attained by the Apéry numbers modulo these primes. This leads us 
to the question of which residue classes are not attained by Apéry-like numbers modulo prime powers $p^{\alpha}$. In particular, are there interesting cases which are not explained by Sections 5 and 6 ?

The second part of congruence (6.4) makes it explicit that every prime $p \equiv 3(\bmod 4)$ divides a term of the Apéry-like sequence (6.3). Is there a similarly explicit result which demonstrates that the Apéry numbers are divisible by infinitely many distinct primes?

Recall that Conjecture 6.3 predicts that the proportion of primes not dividing any Apéry number is $e^{-1 / 2}$. One of the referees raised the question whether there might be a connection between this conjectured proportion and classical divisbility questions of Bernoulli numbers and the notion of regular primes (for instance, C. L. Siegel conjectured that $e^{-1 / 2}$ of all prime numbers are regular).

Another interesting question was suggested by the second referee, who noted that the right-hand side of (6.4) is the $p$-th Fourier coefficient $c_{p}$ of the modular form

$$
\eta(4 z)^{6}:=q \prod_{n=1}^{\infty}\left(1-q^{4 n}\right)^{6}=\sum_{n=1}^{\infty} c_{n} q^{n}, \quad q=e^{2 \pi i z} .
$$

With this observation, a natural question concerning Theorem 6.4 is whether there exists a modular form (with CM?) $f(z)$ whose $p$-th Fourier coefficient is related modulo $p^{2}$ to $A_{\eta}$.

Competing interests

The authors declare that they have no competing interests.

\section{Authors' contributions}

Both authors have contributed to this work in equal parts. Both authors read and approved the final manuscript.

\section{Acknowledgements}

This paper builds on experimental results obtained together with Arian Daneshvar, Pujan Dave and Zhefan Wang during an Illinois Geometry Lab (IGL) project during the Fall 2014 semester at the University of Illinois at Urbana-Champaign (UIUC). The aim of the IGL is to introduce undergraduate students to mathematical research. We wish to thank Arian, Pujan and Zhefan (at the time undergraduate students in engineering at UIUC) for their great work. In particular, their experiments predicted Corollaries 5.1 and 5.2, and provided the data for Table 4, which lead to Conjecture 6.3. We are also grateful to Eric Rowland, who visited UIUC in October 2014, for interesting discussions on Apéry-like numbers and finite state automata, as well as for observing the congruence (6.1).

Moreover, we would like to express our gratitude to Tewodros Amdeberhan, Bruce C. Berndt, Robert Osburn and Wadim Zudilin for many helpful comments and encouragement. Finally, we thank the two referees for their detailed and helpful suggestions.

Received: 3 August 2015 Accepted: 6 January 2016

Published online: 14 February 2016

\section{References}

1. Ahlgren, S: Gaussian hypergeometric series and combinatorial congruences. In: Symbolic computation, number theory, special functions, physics and combinatorics (Gainesville, FL, 1999), volume 4 of Dev. Math., pp. 1-12. Kluwer Acad. Publ., Dordrecht, (2001)

2. Ahlgren, S, Ono, K: A Gaussian hypergeometric series evaluation and Apéry number congruences. J. für die reine und angewandte Mathematik. 2000(518), 187-212 (2000)

3. Almkvist, G, van Straten, D, Zudilin, W: Generalizations of Clausen's formula and algebraic transformations of Calabi-Yau differential equations. Proc. Edinburgh Math. Soc. 54(2), 273-295 (2011)

4. Almkvist, G, Zudilin, W: Differential equations, mirror maps and zeta values. In: Mirror symmetry. V, volume 38 of AMS/IP Stud. Adv. Math., pp. 481-515. Amer. Math. Soc., Providence, RI, (2006)

5. Apéry, R: Irrationalité de $\zeta$ (2) et $\zeta(3)$. Astérisque. 61, 11-13 (1979)

6. Beukers, F: Some congruences for the Apéry numbers. J. Number Theory. 21(2), 141-155 (1985)

7. Beukers, F: Another congruence for the Apéry numbers. J. Number Theory. 25(2), 201-210 (1987)

8. Beukers, F: On Dwork's accessory parameter problem. Math. Z. 241(2), 425-444 (2002)

9. Calkin, N: Factors of sums of powers of binomial coefficients. Acta Arithmetica. 86, 17-26 (1998)

10. Chan, HH, Cooper, S, Sica, F: Congruences satisfied by Apéry-like numbers. Intl. J. Number Theory. 6(1), 89 (2010)

11. Chowla, S, Cowles, J, Cowles, M: Congruence properties of Apéry numbers. J. Number Theory. 12(2), 188-190 (1980)

12. Cooper, S: Sporadic sequences, modular forms and new series for $1 / \pi$. Ramanujan J. 29(1-3), 163-183 (2012)

13. Coster, MJ: Supercongruences. PhD thesis, Universiteit Leiden (1988) 
14. Deutsch, E, Sagan, BE: Congruences for Catalan and Motzkin numbers and related sequences. J. Number Theory. 117(1), 191-215 (2006)

15. Egorychev, GP: Integral representation and the computation of combinatorial sums, volume 59 of Translations of Mathematical Monographs. American Mathematical Society, Providence, RI (1984). Translated from the Russian by H. H. McFadden, Translation edited by Lev J. Leifman

16. Gessel, IM: Some congruences for Apéry numbers. J. Number Theory. 14(3), 362-368 (1982)

17. Krattenthaler, C, Müller, TW: Generalised Apéry numbers modulo 9. J. Number Theory. 147, 708-720 (2015)

18. Lucas, E: Sur les congruences des nombres Eulériens et des coefficients différentiels des fonctions trigonométriques, suivant un module premier. Bull. Soc. Math. France. 6, 49-54 (1878)

19. Mclntosh, RJ: A generalization of a congruential property of Lucas. Amer. Math. Monthly. 99(3), 231-238 (1992)

20. Mellit, A, Vlasenko, M: Dwork's congruences for the constant terms of powers of a Laurent polynomial. Int. J Number Theory (2015). To appear doi:10.1142/S1793042116500184

21. Osburn, R, Sahu, B: Supercongruences for Apéry-like numbers. Adv. Appl. Math. 47(3), 631-638 (2011)

22. Osburn, R, Sahu, B: A supercongruence for generalized Domb numbers. Functiones et Approximatio Commentarii Mathematici. 48(1), 29-36 (2013)

23. Osburn, R, Sahu, B, Straub, A: Supercongruences for sporadic sequences. Proc. Edinburgh Math Soc (2015). To appear doi:10.1017/S0013091515000255

24. Poorten, AVD: A proof that Euler missed... Apéry's proof of the irrationality of $\zeta$ (3). Math. Intelligencer. 1(4), 195-203 (1979)

25. Rowland, E, Yassawi, R: Automatic congruences for diagonals of rational functions. J. de Théorie des Nombres de Bordeaux. 27(1), 245-288 (2015)

26. Samol, K, van Straten, D: Dwork congruences and reflexive polytopes. Annales mathématiques du Québec. 39(2), 185-203 (2015)

27. Stienstra, J, Beukers, F: On the Picard-Fuchs equation and the formal Brauer group of certain elliptic K3-surfaces. Mathematische Annalen. 271(2), 269-304 (1985)

28. Straub, A: Multivariate Apéry numbers and supercongruences of rational functions. Algebra Number Theory. 8(8), 1985-2008 (2014)

29. Zagier, D: Integral solutions of Apery-like recurrences. In: Harnad, J, Winternitz, P (eds.) Groups and symmetries. From Neolithic to John McKay, volume 47. American Mathematical Society, Providence, Rl, (2009)

\section{Submit your manuscript to a SpringerOpen ${ }^{\circ}$ journal and benefit from:}

- Convenient online submission

- Rigorous peer review

- Immediate publication on acceptance

- Open access: articles freely available online

- High visibility within the field

- Retaining the copyright to your article

Submit your next manuscript at $\gg$ springeropen.com 